ARTICLE

\title{
Structural basis for distinct inflammasome complex assembly by human NLRP1 and CARD8
}

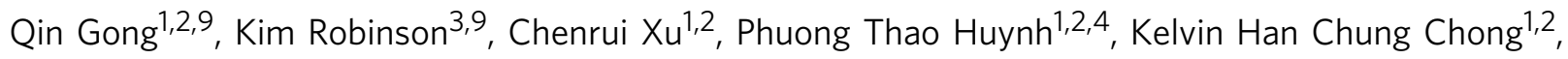
Eddie Yong Jun Tan'1,2, Jiawen Zhang, ${ }^{1,2}$, Zhao Zhi Boo ${ }^{1,2}$, Daniel Eng Thiam Teo ${ }^{5}$, Kenneth Lay (1) ${ }^{5}$, Yaming Zhang ${ }^{1,2}$, John Soon Yew Lim ${ }^{3}$, Wah Ing Goh ${ }^{3}$, Graham Wright ${ }^{5}$, Franklin L. Zhong $\mathbb{D}^{3,4,5 凶}$,

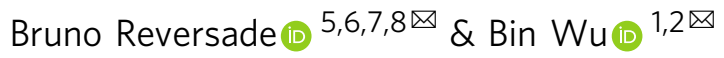

Nod-like receptor (NLR) proteins activate pyroptotic cell death and IL-1 driven inflammation by assembling and activating the inflammasome complex. Closely related sensor proteins NLRP1 and CARD8 undergo unique auto-proteolysis-dependent activation and are implicated in auto-inflammatory diseases; however, their mechanisms of activation are not understood. Here we report the structural basis of how the activating domains (FIINDUPA-CARD) of NLRP1 and CARD8 self-oligomerize to assemble distinct inflammasome complexes. Recombinant FIINDUPA-CARD of NLRP1 forms a two-layered filament, with an inner core of oligomerized CARD surrounded by an outer ring of FIINDUPA. Biochemically, self-assembled NLRP1-CARD filaments are sufficient to drive ASC speck formation in cultured human cellsa process that is greatly enhanced by NLRP1-FIINDUPA which forms oligomers in vitro. The cryo-EM structures of NLRP1-CARD and CARD8-CARD filaments, solved here at $3.7 \AA$, uncover unique structural features that enable NLRP1 and CARD8 to discriminate between ASC and pro-caspase-1. In summary, our findings provide structural insight into the mechanisms of activation for human NLRP1 and CARD8 and reveal how highly specific signaling can be achieved by heterotypic CARD interactions within the inflammasome complexes.

\footnotetext{
${ }^{1}$ School of Biological Sciences, Nanyang Technological University, Singapore 637551, Singapore. ${ }^{2}$ NTU Institute of Structural Biology, Nanyang Technological University, Singapore 636921, Singapore. ${ }^{3}$ Skin Research Institute (SRIS), Agency of Science Technology and Research (A*STAR), 8A Biomedical Grove, \#06-06 Immunos, 138648 Singapore, Singapore. ${ }^{4}$ Lee Kong Chian School of Medicine, Nanyang Technology University, 11 Mandalay Road, 308232 Singapore, Singapore. ${ }^{5}$ Institute of Molecular and Cell Biology, Agency of Science Technology and Research (A*STAR), 61 Biopolis Dr, 138673 Singapore, Singapore. ${ }^{6}$ Genome Institute of Singapore, Agency of Science Technology and Research (A*STAR), 8A Biomedical Grove, \#06-06 Immunos, 138648 Singapore, Singapore. ${ }^{7}$ Department of Paediatrics, Yong Loo Lin School of Medicine, National University of Singapore, 10 Medical Dr, 117597 Singapore, Singapore. ${ }^{8}$ The Medical Genetics Department, School of Medicine (KUSoM), Koç University, 34010 Istanbul, Turkey. ${ }^{9}$ These authors contributed

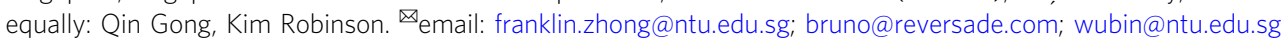


$\mathrm{N}$ od-like receptor (NLR) proteins are cytosolic sensors and activators of the innate immune response. Many NLR proteins nucleate the "inflammasome"-a large macromolecular complex that triggers inflammatory cell death and cytokine secretion in response to microbial or danger-associated ligands. Like many other supramolecular assemblies in the human innate immune system ${ }^{1,2}$, the inflammasome complex relies on homotypic and heterotypic interactions between the death domains (DDs) to initiate, amplify, and propagate pyroptotic signaling ${ }^{3,4}$. Inflammasome sensor NLRs can be grouped into two categories based on their DDs: PYRIN domain (PYD) and caspase activation and recruitment domain $(\mathrm{CARD})^{5-7}$, both domains were found to form filamentous complexes to initiate the inflammasome formation. Recently, we and others have expanded the repertoire of CARD-based human inflammasome sensors to include NLRP1 and a related receptor, CARD8 ${ }^{8-11}$, both of which are implicated in human autoinflammatory dis-

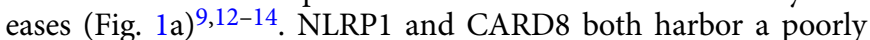
defined auto-proteolytic domain known as FIIND, whose autocleavage is required for inflammasome activation ${ }^{15,16}$. The requirement of the adaptor protein ASC has also been a matter of debate for CARD-containing sensor proteins such as NLRP1 and NLRC4 ${ }^{17}$. Very recently, human CARD8, which is absent from rodents, was found to bypass ASC altogether and activate caspase- 1 directly ${ }^{18}$.

In this study, we investigate the structural basis of how the auto-proteolysis-dependent, activating domains of human NLRP1 and CARD8 (FIINDUPA_CARD) trigger inflammasome assembly and activation. We demonstrate that FINDUPA and CARD domains play non-redundant roles in NLRP1 activation: NLRP1-CARD is sufficient to form filaments in vitro, trigger ASC speck formation and activate pyroptosis in human cells, albeit with limited efficiency. NLRP1-FIIND ${ }^{\text {UPA }}$ does not interact with ASC directly, but dramatically lowers the threshold of NLRP1CARD oligomerization and filament formation. Mechanistically, stand-alone FIIND ${ }^{\mathrm{UPA}}$ forms ring-like oligomers, whereas recombinant FIIND UPA_CARD forms a two-layered filament, with oligomerized CARD as the inner core around which a FIINDUPA ring spirals. Further analysis reveals that a 9-22amino-acid linker between FIIND and CARD contributes to the distinct helical symmetry of the NLRP1- and CARD8- FIIN$\mathrm{D}^{\mathrm{UPA}}$-CARD two-layered filaments. By solving the cryo-EM structures NLRP1- and CARD8-CARD core filaments at $3.7 \AA$, we map the precise structural motifs that mediate heterotypic interactions between the CARD domains of NLRP1, CARD8, ASC, and CASP1. This analysis provides a structural explanation as to why activated NLRP1 avidly triggers ASC speck formation, whereas CARD8 directly activates pro-caspase-1. In conjunction with three complementary ${ }^{19-21}$ preprint manuscripts published while this manuscript was in revision, our findings unveil the structural basis for NLRP1 and CARD8 inflammasome assembly and suggest that intrinsic structural properties of the CARD domain can determine the specificity of inflammasome signaling.

\section{Results}

NLRP1-FIIND UPA domain facilitates NLRP1-CARD oligomerization. Previously, we and others have demonstrated that Cterminal auto-cleavage fragments of NLRP1 and CARD8

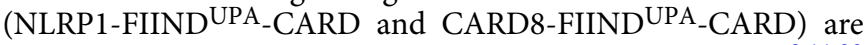
sufficient to drive pyroptosis in cultured human cells ${ }^{9,11,22}$ (Fig. 1a). To further map the exact role of FIIND ${ }^{\text {UPA }}$, we utilized the widely used "ASC-speck" assay in HEK239T cells expressing GFP-tagged full-length ASC (293T-ASC-GFP). Overexpressed NLRP1-CARD is sufficient to induce ASC-GFP specks, but does so much less readily as compared with the FIINDUPA_CARD fragment, in agreement with previous findings ${ }^{9,22}$. This difference is especially notable at lower levels of protein expression (Fig. 1b, 50 ng; Fig. 1c, lane 3 and 6). At limiting protein expression levels, $\sim 90 \%$ of NLRP1-CARD exists in the monomeric form (Fig. $1 \mathrm{~d}$, lane 2 and 3), with the rest as higher-molecular weight oligomers, in agreement with its low but detectable activation capacity. In contrast, FIINDUPA_CARD, at comparable expression levels, exists mostly as high-molecular-weight oligomeric species (Fig. 1d, lane 1 vs. 2 and 3). Thus, although NLRP1-CARD is sufficient to induce ASC specks when oligomerized at elevated concentrations, the presence of FIIND ${ }^{\mathrm{PPA}}$ domain strongly enhances its activation by lowering the threshold of oligomerization.

Next, we examined recombinant NLRP1-CARD, FIIND UPA CARD, and a 1:1 molar mixture of FIIND ${ }^{\text {UPA }}$ with CARD using negative stain-electron microscopy (EM). In agreement with the native PAGE results in $293 \mathrm{~T}$ cells, NLRP1-FIINDUPA lowered the concentration requirement for CARD oligomerization by at least 50-fold (Fig. 1e, f), but only when expressed along with CARD as a single polypeptide (Fig. 1f). NLRP1-CARD, either in isolation or incubated with FIIND ${ }^{\mathrm{UPA}}$ as a separate polypeptide, was unable to form ordered oligomers at concentrations $<\sim 200 \mu \mathrm{M}$, but did appear as long ordered oligomers at concentrations at $500 \mu \mathrm{M}$ or above (Fig. 1e, right, Fig. 1f). These results suggest that NLRP1-CARD has a limited but detectable propensity for self-oligomerization. The FIINDUPA domain, akin to a "built-in" catalyst, strongly lowers the threshold for NLRP1CARD oligomerization.

To seek direct evidence for this model, we purified the following NLRP1 fragments: FIIND UPA_CARD, full-length FIIND-CARD (FIIND ${ }^{\mathrm{fl}-C A R D), ~ F I I N D ~}{ }^{\text {UPA }}$, and CARD alone (Supplementary Fig. 1). Both HEK293T cell-derived and bacteriaderived FIINDUPA-CARD formed long heterogenous filaments $\sim 14-15 \mathrm{~nm}$ in diameter (Supplementary Fig. 1a, b, e), but not FIIND ${ }^{\text {UPA }}$ alone (Supplementary Fig. 1c), or FIIND ${ }^{\mathrm{fl}-\mathrm{CARD}}$ (Supplementary Fig. 1d). In agreement with its detectable oligomerization capacity in $293 \mathrm{~T}$ cells, recombinant NLRP1CARD also formed filaments, similar to CARD domains found in other innate immune complexes. As expected, these filaments were visibly thinner than those formed by FIIND ${ }^{\text {UPA_CARD }(\sim 8}$ $\mathrm{nm}$ in diameter) (Supplementary Fig. 1b, e).

We next set out to solve the structures of NLRP1-FIIND UPA CARD and the equivalent activating fragment of CARD8. Detailed construct optimization was conducted to determine the roles of linkers and exact domain boundaries to facilitate structure determination (Supplementary Fig. 2). Truncation analysis demonstrated that the first 11 residues of NLRP1FIINDUPA (a.a. 1213-1223), 11 residues from the linker region (a.a.1360-1370), and the last eight amino acids of CARD (1466-1473) were dispensable for NLRP1-FIIND ${ }^{\text {UPA }}$-CARD to induce ASC-GFP speck formation and oligomer formation (Supplementary Fig. 2a-c). However, these shorter constructs did not improve filament homogeneity in vitro; hence the full NLRP1-FIIND UPA_CARD filaments (a.a. 1213-1473) were used for subsequent structural studies (Supplementary Fig. 1a, e). Under negative stain EM, multiple 2D classes with distinct rotational symmetries were present with various diameters and helical settings (Fig. 1g). In all, 3D projection of the most abundant $15 \mathrm{~nm} 2 \mathrm{D}$ class yielded a helical filament with 11-12 identical subunits per turn and $\sim 5.6 \mathrm{~nm}$ per helical rise, which is coincidentally similar to the rise of NLRC4 full-length disc-like complexes $^{23}$. Notably, when examined using differing contour levels, this NLRP1-FIINDUPA_CARD filament class was found to consist of a denser inner core filament with a diameter of $\sim 8 \mathrm{~nm}$ (Fig. 1h). Surrounding this inner core is a discrete outer layer that extends to $15 \mathrm{~nm}$ across the center decorated with distinct "blobs" 

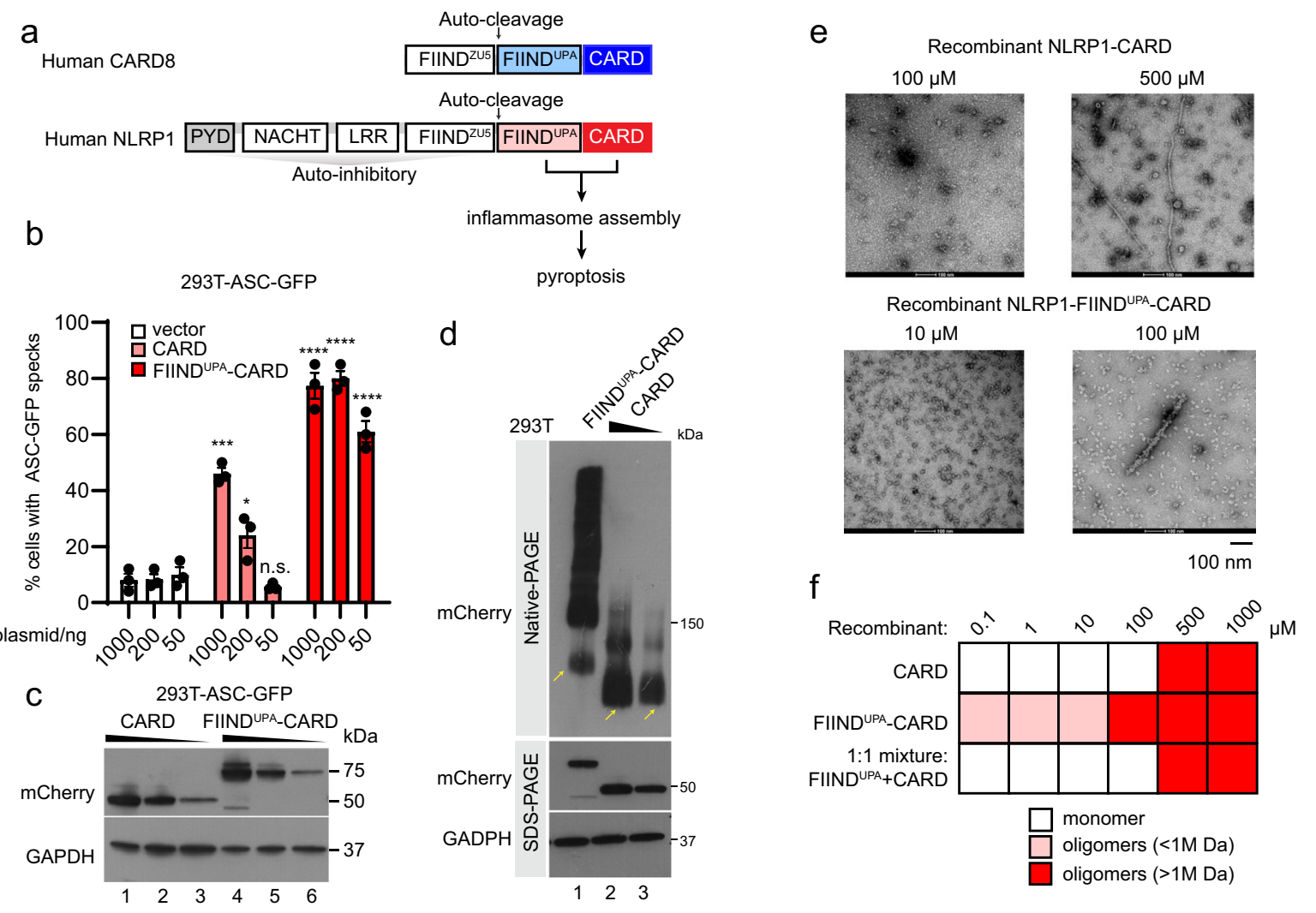

g Representatitive 2D classification of recombinant $\quad$ h
h 3D density of helical reconstructed $15 \mathrm{~nm}$ class and a corn on the cob with similar helical symetry side view
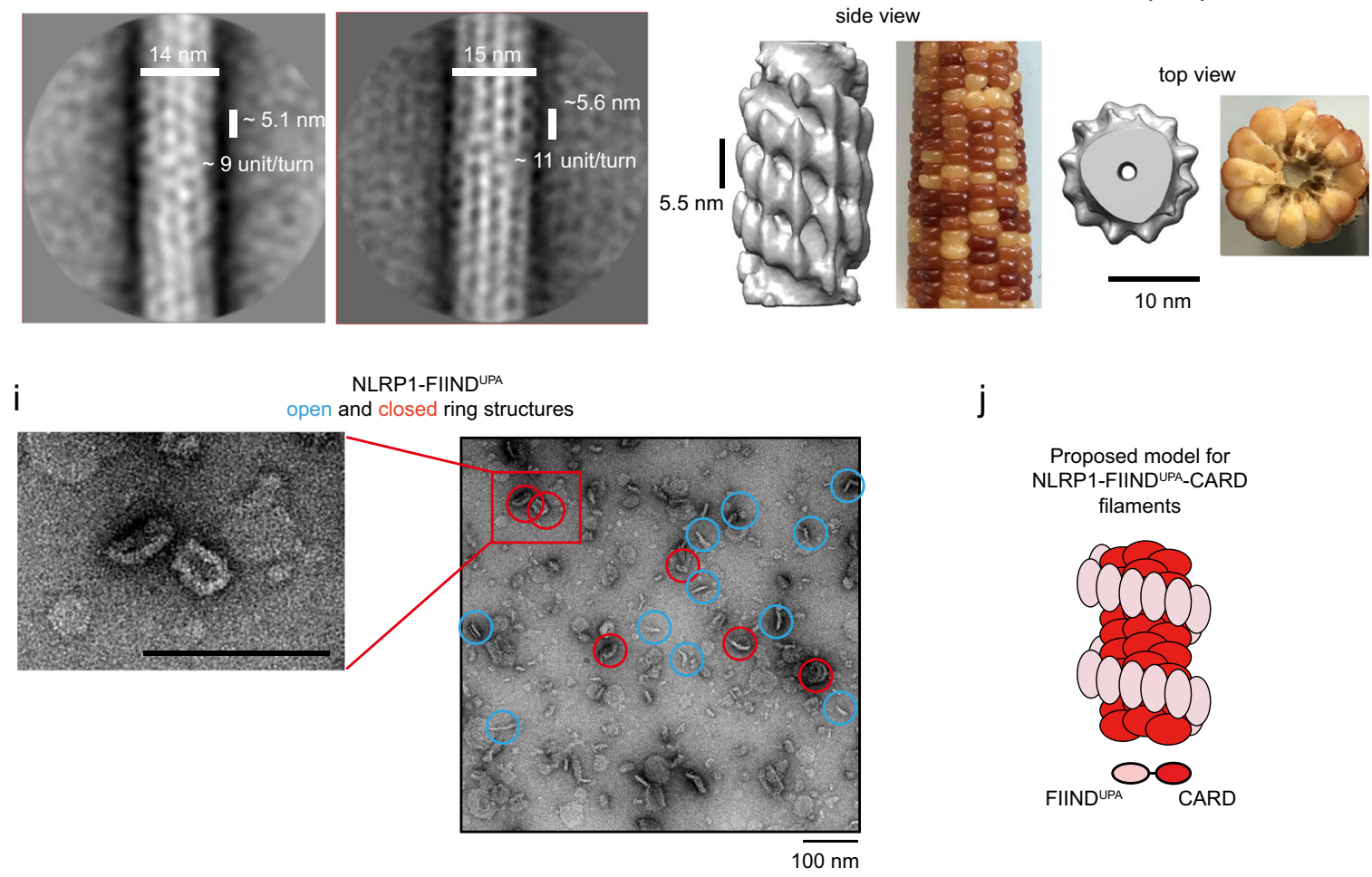

of protruding density, which resembles a corn cob (Fig. 1h). We were fascinated to identify such a "two-layer" architecture for a FIIND-containing inflammasome sensor. It is reminiscent of activated NLRC4 and NLRP6 ${ }^{24,25}$, where the nucleotide-binding domain (NBD) pre-oligomerizes to bring the CARD or PYDs into close proximity. The observed dimensions of the inner core filament, akin to the stall of the corn cob are consistent with those of the recombinant filaments composed of CARD alone (Supplementary Fig. 1b, $8 \mathrm{~nm}$ in diameter, see below). The most likely explanation is that the FIIND ${ }^{\mathrm{UPA}_{-}}$-CARD oligomers consist of an inner helical filament of oligomerized CARDs surrounded by a FIIND ${ }^{\text {UPA }}$ spiral facing outward (Fig. 1h). As further 
Fig. 1 Human NLRP1 inflammasome complexes. a Domain organizations of human NLRP1, CARD8. Death domains (PYD and CARD) are highlighted in blue or red. $\mathbf{b}$ Percentage of ASC-GFP specks induced by different amounts of mCherry-tagged NLRP1-FIINDUPA-CARD (red) or mCherry-tagged NLRP1CARD (light red) in HEK293T-ASC-GFP cells. Cells were fixed 24 hours after transfection for fluorescence microscopy. Data are presented as mean values \pm SD. $P$ value was calculated with one-way ANOVA, $n=3$ biological replicates. ${ }^{\star \star} /{ }^{\star \star} /{ }^{\star \star \star \star \star \prime}$ indicates $P$ value $<0.05,0.01$, and 0.0001 , correspondingly. c Corresponding western blot of mCherry-tagged NLRP1 constructs in Fig. 1b. d Blue-native PAGE blot of mCherry-tagged NLRP1FIINDUPA_CARD and mCherry-tagged NLRP1-CARD. Yellow arrows, monomeric species. e Representative negative stain EM images recombinant NLRP1FIINDUPA-CARD and NLRP1-CARD at various concentrations. $\mathbf{f}$ A summary of oligomer formation of various NLRP1 constructs at various concentrations. $\mathbf{g}$ Two of the best 2D class images with clearly distinguishable features of recombinant NLRP1-FIINDUPA-CARD filamentous complex, from negative stain EM analysis. $\mathbf{h}$ A side view and a top view of the 3D density model at estimated 20-30 $\AA$ resolution (after 3D refinement using RELION) generated based on particles extracted from the $15 \mathrm{~nm} 2 \mathrm{D}$ class. A similar heterogenous helical symmetry can be found in some corn cobs. $\mathbf{i}$ Negative EM images of SUMOtagged NLRP1-FIINDUPA. The ring-complexes are $-20 \mathrm{~nm}$ in size. $\mathbf{j}$ Proposed model for NLRP1 FIINDUPA-CARD oligomers.

evidence for this model, recombinant SUMO-tagged NLRP1FIIND ${ }^{\mathrm{UPA}}$ spontaneously formed ring-shaped or linear oligomers of heterogeneous stoichiometries in vitro. These closed "rings" had a minimum diameter of $\sim 17 \mathrm{~nm}$ (Fig. 1i, red circles). Accommodating the SUMO tag, this is consistent with the dimensions of the outer periphery of the NLRP1-FIIND ${ }^{\text {UPA }}$ CARD filament, similar to the kernels of the corn cob (Fig. 1h-j). Similar biochemical analysis of the FIIND UPA_CARD and FIIND UPA domains from CARD8 revealed that CARD8FIINDUPA most likely functions in a similar way as those of NLRP1. Recombinant CARD8-FIIND ${ }^{\text {UPA }}$ also formed oligomers, although the oligomer architecture appears to be more heterogeneous in nature (Supplementary Fig. $3 \mathrm{a}-\mathrm{c}$ ). To improve the homogeneity and/or stability of the CARD8-FIINDUPA_CARD filaments, we engineered a 14-residue linker between the UPA and CARD domain (Supplementary Fig. 2d-h), which did not affect the ability of CARD8-FIINDUPA_CARD to induce CASP1CARD oligomerization (Supplementary Fig. $2 \mathrm{f}-\mathrm{h}$ ). Negative stain EM images of both wild-type and 14 a.a. linker constructs were pooled together, filaments were picked and analyzed by $2 \mathrm{D}$ classification (Supplementary Fig. 3d). A clear two-layer filament feature was seen after enhancing the contrast of the $2 \mathrm{D}$ class images (Supplementary Fig. 3d, right). We then carried out 3D classification of selected 2D classes without pre-defining any helical symmetry (Supplementary Fig. 3e, f). Despite the significant heterogeneity, the overall architecture of CARD8FIINDUPA_CARD resembles that of NLRP1-FIINDUPA_CARD (Supplementary Fig. 3f). In further support of the two-layer filament architecture, the top view of the CARD8-FIINDUPA CARD 3D class images, after applying the helical symmetry, closely resembled that of untagged CARD8-FIIND ${ }^{\text {UPA-CARD }}$ after refolding (Supplementary Fig. 3g, h). Hence, despite being less organized, CARD8-FINDUPA-CARD also forms two-layered filaments with similar dimensions and arrangement as NLRP1FIINDUPA_CARD (Supplementary Fig. 3h, i). These findings offer a structural explanation for the necessity for the FIINDUPA domain in aiding CARD oligomerization in both NLRP1 and CARD8. Once triggered, NLRP1-FIINDUPA and CARD8FIINDUPA pre-oligomerizes to bring the respective CARD domains in close proximity to activate downstream inflammasome signaling.

Cryo-EM structures of NLRP1- and CARD8-CARD filaments: the inner core of the FIIND ${ }^{\text {PA }}$-CARD filaments. It was recently reported that human NLRP1 and CARD8, long thought to be functional paralogues, can give rise to distinct signaling outcomes: while NLRP1 avidly triggers ASC speck formation, CARD8 can directly activate pro-caspase-1 without the need for $\mathrm{ASC}^{18}$. The mechanistic basis for this disparity is currently unclear. Considering our finding demonstrating that NLRP1CARD, once oligomerized, is sufficient to activate downstream inflammasome signaling (Fig. 1b), we hypothesized that this difference could be owing to the intrinsic structural differences between NLRP1-CARD and CARD8-CARD. To test this, we solved the structures of recombinant NLRP1-CARD and CARD8CARD filaments using cryo-EM, both at $3.7 \AA$ with Gold standard FSC $=0.143$, without masking (Fig. 2a-i, Supplementary Fig. 4a and Supplementary Table 1). NLRP1-CARD filaments are relatively flexible and adopt multiple helical organizations in vitro, as evidenced by heterogeneous $2 \mathrm{D}$ and $3 \mathrm{D}$ classes observed during structural analysis using RELION 3.0 (Supplementary Fig. $4 \mathrm{~b})^{26}$. One out of four observed 3D class conformations of NLRP1-CARD filaments was determined to be suitable for high-resolution structural determination ${ }^{27}$ (Supplementary Fig. 4c, Fig. 2b-e). Trimming of the last eight a.a. (1466-1473) helped stabilize the homogenous form of NLRP1CARD single filament (Supplementary Fig. 1e). This conformation was subsequently verified to be a signaling-competent confirmation based on functional experiments using 293T-ASC-GFP cells (see below). Importantly, the resolved CARD filament density fits snugly into the lower resolution model of NLRP1FIINDUPA_CARD filament based on negative stain EM, matching the shape and dimensions of the inner layer almost perfectly (Fig. 2e). Similar to NLRP1-CARD, recombinant CARD8-CARD also formed $\sim 8 \mathrm{~nm}$-wide filaments, but these filaments are more rigid and adopt a single conformation (Fig. $2 \mathrm{f}-\mathrm{h}$, and Supplementary Fig. 5a, b). The final polished densities demonstrated clear $\alpha$-helix pitch and supported the unambiguous assignment of all bulky side chains (Fig. 2d, i, Supplementary Fig. 5a-d) in both filaments.

NLRP1-CARD and CARD8-CARD filaments display similar helical parameters $(5.36 \AA$ and -100.8 degree for NLRP1, and $5.409 \AA$ and -99.16 degree for CARD8), suggesting a similar mode of assembly from the respective monomers (Fig. 2d, i). Both filaments also have clearly demarcated Type I-III interfaces that are characteristic of oligomerized DD (Fig. 2j-k, Supplementary Fig. $5 c, d)^{2,24,28,29}$. The definition of the Type I-III interface follows the naming convention first used in describing the MyDDosome complex ${ }^{2}$, and subsequently widely adopted in describing DD filaments, such as MAVS-CARD. When positioning the first alpha-helix of CARD domain downward, and the sixth alpha-helix upward, the monomer on top is called "a" monomer, and all three neighboring monomers are called "b" monomers. From left to right, each "b" monomers have a unique interaction interface with "a" monomer, and from left to right, named as Type I, Type II, and Type III interfaces, respectively. The corresponding surfaces on "a" monomer are Type Ia, IIa, and IIIa surfaces, and the surfaces on the three " $b$ " monomers are type $\mathrm{Ib}$, IIb, and IIIb surfaces. All surface interactions within DD filaments could be grouped into one of these types of interfaces (Fig. 2d, i). As compared with other DD complexes, the Type II interfaces in NLRP1- and CARD8-CARD filaments display stronger and more-prominent electrostatic interactions than Type I and Type III (Fig. 2j, k). This is particularly notable in 
NLRP1-CARD filament

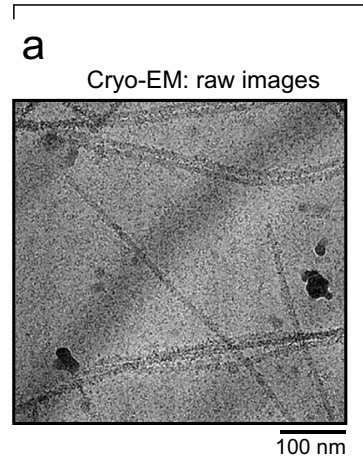

b

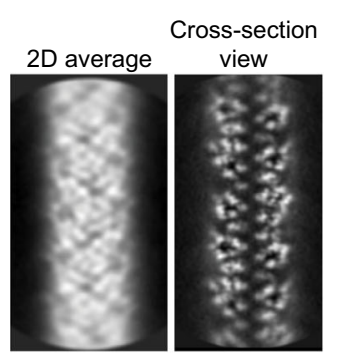

Top view

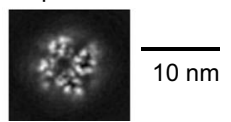

d e

$3 \mathrm{D}$ reconstruction
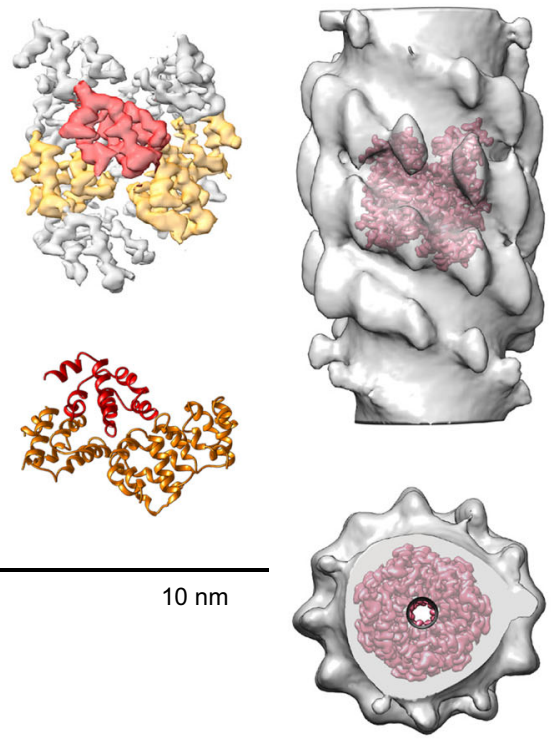

$10 \mathrm{~nm}$
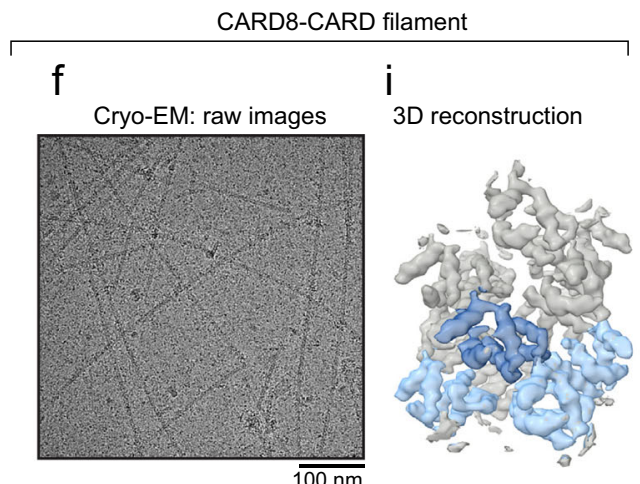

$\mathrm{h}$

g
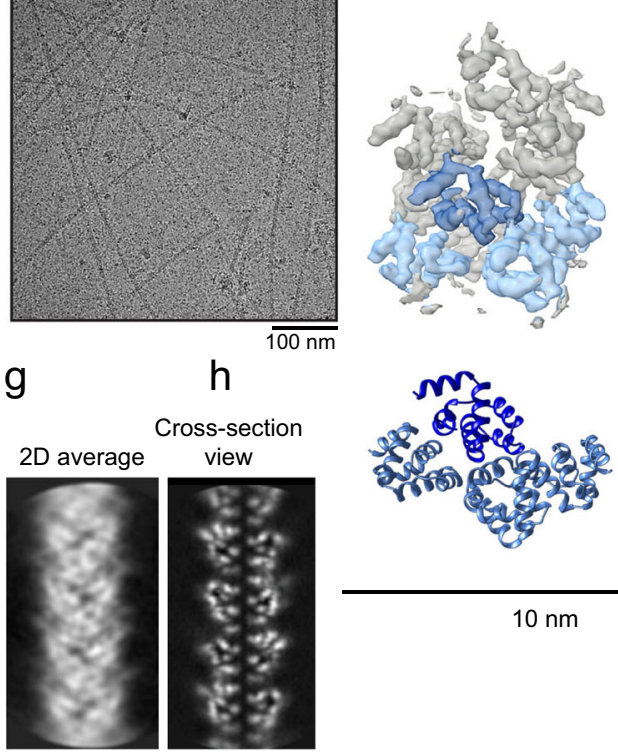

$10 \mathrm{~nm}$

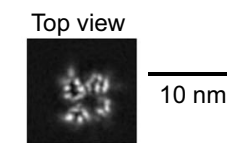

k

j

NLRP1-CARD filament

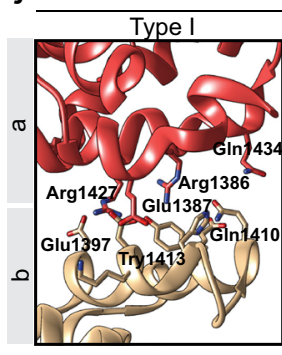

I

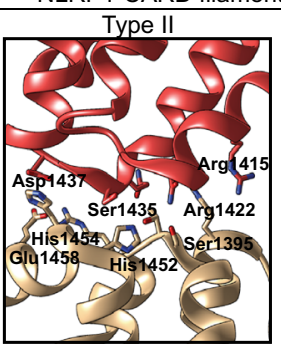

293T-responder: ASC-GFP

inducer: NLRP1-FIIND UPA_CARD-mCherry

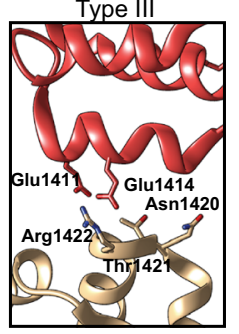

$\mathrm{k}$

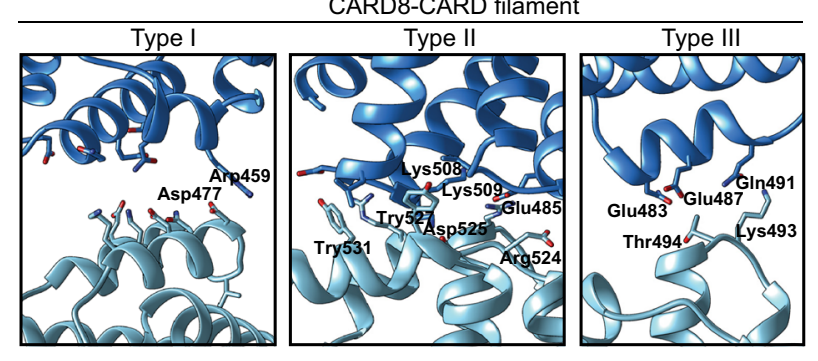

$$
\begin{aligned}
& \text { 293T-responder: CASP1-CARD-GFP } \\
& \text { inducer: CARD8-CARD-mCherry }
\end{aligned}
$$

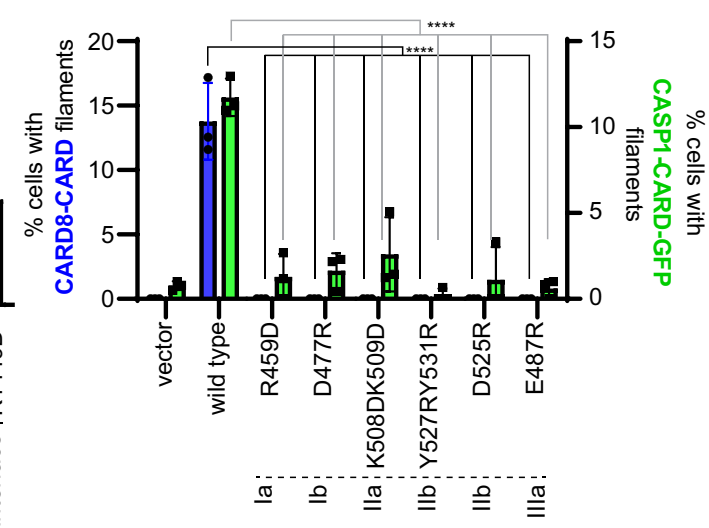

CARD8-CARD filaments, where Type Ia and Ib surfaces are separated from each other (Fig. 2j, k). To validate these structures functionally, predicted type I-III interface residues were mutated by site-directed mutagenesis. For both filaments, missense mutations of Type I-III interface residues abrogated filament assembly as well as their ability to nucleate downstream ASC-
GFP or CASP1-CARD-GFP specks/filaments (Fig. 2l, m), whereas non-interface surface-exposed residues have less effect. These results further prove that CARD oligomerization is a prerequisite for NLRP1 and CARD8-driven inflammasome activation. A notable exception is the type Ia interface of NLRP1, where two of the four mutations, E1387R and Q1434R, did not affect ASC-GFP 
Fig. 2 Cryo-EM structures of NLRP1-CARD and CARD8-CARD filaments. a Cryo-EM images of recombinant NLRP1-CARD filaments. b 2D average of NLRP1-CARD filaments. c Cross-section and top view of NLRP1-CARD filaments. d Helical reconstructed NLRP1-CARD complex density and molecular models. Adjacent NLRP1-CARD monomers are colored in light red ("a" monomer) and orange ("b" monomers). e Atomic resolution NLRP1-CARD filament density fit into the inner layer density of NLRP1-FIINDUPA-CARD shown in Fig. Th. $\mathbf{f}$ Cryo-EM raw images of recombinant CARD8-CARD filaments. $\mathbf{g}$ 2D average of CARD8-CARD filaments. $\mathbf{h}$ Cross-section and top view of CARD8-CARD filaments. $\mathbf{i}$ Helical reconstructed CARD8-CARD complex density and molecular models. Adjacent CARD8-CARD monomers are colored in blue ("a" monomer) and light blue ("b" monomers). j Type I-III interfaces in NLRP1CARD filaments. k Type I-III interfaces in CARD8-CARD filaments. I Mutagenesis of critical NLRP1-CARD interface residues and their effects on NLRP1FIIND UPA-CARD-mCherry to induce ASC-GFP specks in 293 T cells. Data are presented as mean values \pm SD. $P$ value was calculated with One-way

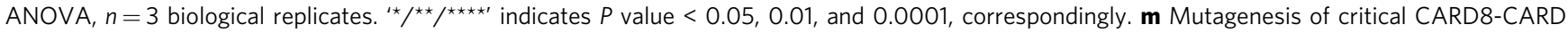
interface residues and their effects on CARD8-CARD-mCherry filament formation (blue) and CASP1-CARD-GFP oligomerization in 293 T cells (green).

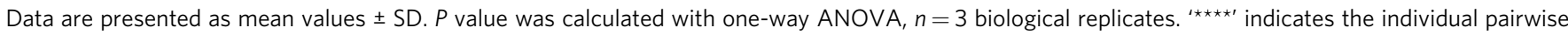
one-sided statistical analysis of wild-type and mutant has a $P$ value $<0.0001$.

speck formation in HEK293T cells despite partially reducing NLRP1-CARD filament assembly in vitro (Fig. 2l, Supplementary Fig. 4d). We postulate that the Type Ia (bottom-facing) interface of NLRP1 is predominantly involved in NLRP1-CARD selfoligomerization, rather than NLRP1-ASC interaction.

FIIND ${ }^{\text {UPA }}$ organization in the outer layer of FIIND ${ }^{\text {UPA_CARD }}$ filament. Next, we carried out scanning mutagenesis to define the structural determinants for NLRP1-FIINDUPA selfoligomerization. In total, nine mutations aimed at disrupting bulky sidechain residues were introduced into a SUMO-NLRP1FIIND ${ }^{\mathrm{UPA}}$ construct based on the homologous UPA structure (PDB 3G5B) ${ }^{30}$. The P1278A P1279G (PP1278AG, colored in red) mutant had the strongest effect at disrupting the oligomerization of SUMO-NLRP1-FIIND ${ }^{\text {UPA }}$ on native gel (Fig. 3a), whereas four other mutants (colored in orange), R1240D, RK1260DD, R12785D, and F1320A more subtly reduced NLRP1-FIINDUPA oligomerization. In agreement with the necessity for the UPA domain to facilitate NLRP1 activation, all oligomerization mutants abrogated the ability for NLRP1-FIINDUPA-CARD to induce ASC-GFP specks, with PP1278AG having the strongest effect (Fig, 3b, c). Taken together, these findings suggest that NLRP1-FIIND UPA employs one large hydrophobic surface patch centered around a.a. 1278-1279 (red) to self-oligomerize in a "side-by-side" arrangement (Fig. 3d), giving rise to the outer ring (Fig. 3e) that wraps around the CARD core in the FIIND UPA CARD filaments (Fig. 1j). This organization closely resembles the FIINDUPA-dimer structures seen in two recently reported NLRP1-DPP9 complexes ${ }^{19,21}$. A similar architecture is likely true for CARD8 (Fig. 3g). This model also explains the necessity for the interdomain linker between UPA and CARD for optimal NLRP1 and CARD8 filament formation (Supplementary Fig. 3c). A zoomed-in view reveals that the optimal linker between UPA and CARD is $\sim 25 \sim 40 \AA$ (Fig. 3f). Shorter linkers would incur spatial constraint and prevent filament extension, whereas longer linkers would produce an outer layer that is too far away from the inner core, potentially destabilizing the FIINDUPA_CARD complex. Multiple sequence alignment confirmed that 9-20 a.a. linker length is true for both human and rodent NLRP1 homologs (Supplementary Fig. 2d), suggesting that arrangement of the FIIND and CARD domains are conserved.

NLRP1 and CARD8-CARD oligomers have distinct surface charge distributions despite similar helical symmetry. Despite near-identical helical parameters, NLRP1-CARD and CARD8CARD filaments have remarkably different surface charge distributions (Fig. 4a-f), that are not suggested by their monomer structures alone ${ }^{31,32}$. NLRP1-CARD filaments demonstrate a balanced charge distribution on its axial surface, similar to previously published DD complexes (Fig. $4 \mathrm{a})^{2,4,33}$. By contrast, in CARD8-CARD filaments, the positively charged patches on the monomer are mostly buried inside the filamentous oligomer, exposing a predominantly negative surface (Fig. 4b). The density from the Type I interface, which has a prominent role in supporting the oligomerization of other CARD helical complexes, does not seem to play a major role in the observed surface density in either complex. Instead, the surface charge distribution is largely due to unusually strong Type II interface densities in both filaments. In the NLRP1-CARD complex, two positively charged histidine residues (His1454 and His1452) extend from the Type IIb surface and interact with negatively charged residues, Asp1437 and Ser1435 on the Type IIa surface (Fig. 4c). In contrast, the CARD8 Type IIb surface is partially negatively charged (Tyr531, Tyr527, and Asp525), whereas the Type IIa surface is positive (Lys508, Lys509, and Leu512) (Fig. 4d). These distinct arrangements of the Type II interfaces are a direct result of the subtle differences (2 degree in helical rotation and $\sim 15$ degree of monomer rotational position) in their helical symmetries, and therefore not easily predicted from the amino-acid sequence differences between NLRP1 and CARD8. Interestingly, the top surface of NLRP1-CARD closely resembles the charge distributions of ASC-CARD oligomer top surface (ASC-CARD filament solved in-house, PDB-6K99, EMD-9947, Fig. 4e, g, left), and CARD8 top surface is more similar to CASP1-CARD top surface (Fig. 4f, h, left). Hence, NLRP1-CARD top surface is predicted to preferentially interact with the bottom surface of ASC-CARD (compare Fig. 4e, g, right), whereas the CARD8-CARD top surface is expected to preferentially interact with the CASP1-CARD bottom surface (compare Fig. 4f, h, right). These structural findings offer a potential explanation for the differing affinity and requirement for ASC between NLRP1 and CARD8, which was recently inferred on the basis of cellular experiments.

Structural basis for the distinct downstream CARD preference between NLRP1 and CARD8. To identify the precise structural determinants that are responsible for ASC vs. pro-caspase-1 binding, we focused on the Type II surfaces of the respective CARD oligomers. This surface has a prominent role in the observed differences in surface charge distribution between NLRP1-CARD and CARD8-CARD filaments (Figs. 2j, k, 4a-f). We hypothesized that the Type II junctions are not only important for homotypic assembly between identical CARD subunits, but also heterotypic interactions between "sensor" CARDs (NLRP1 and CARD8) and downstream effector CARDs (ASC and CASP1). To test this, we first identified the Type II interfaces in published ASC-CARD and CASP1-CARD filament structures (Supplementary Fig. 6a-e, Supplement Table 1). In both structures, we noted a similar internal density bridge on Type II interface as what was observed in NLRP1-CARD and CARD8-CARD complexes (compare Fig. 5a, b to Fig. 4c, d), 


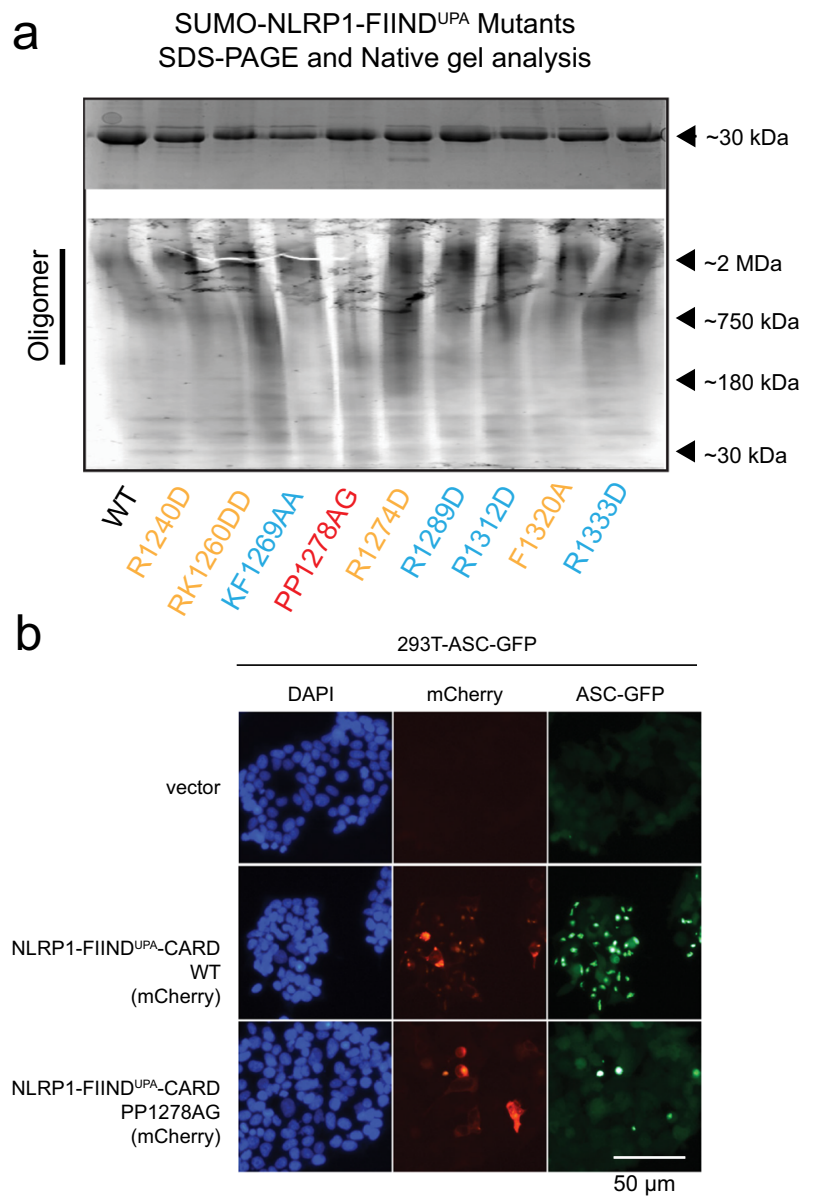

e

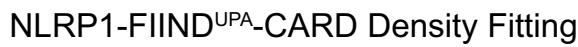
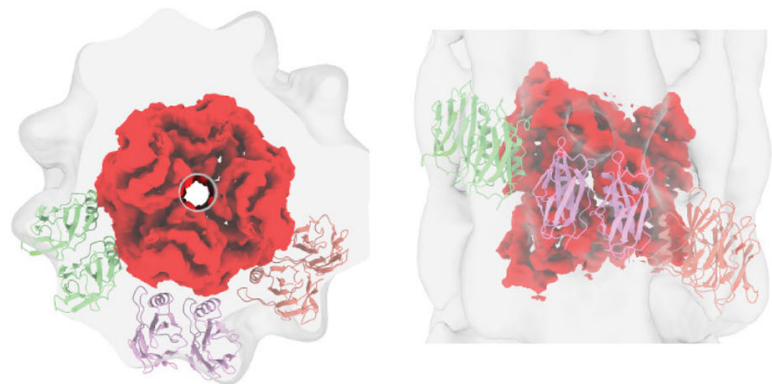

g
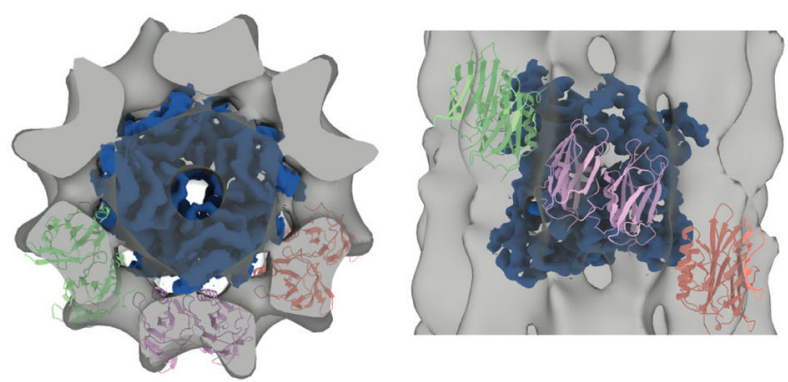

C

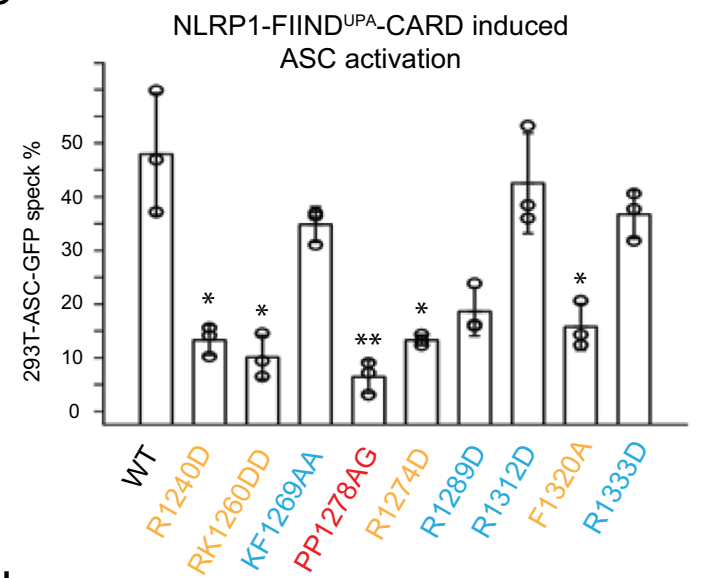

d
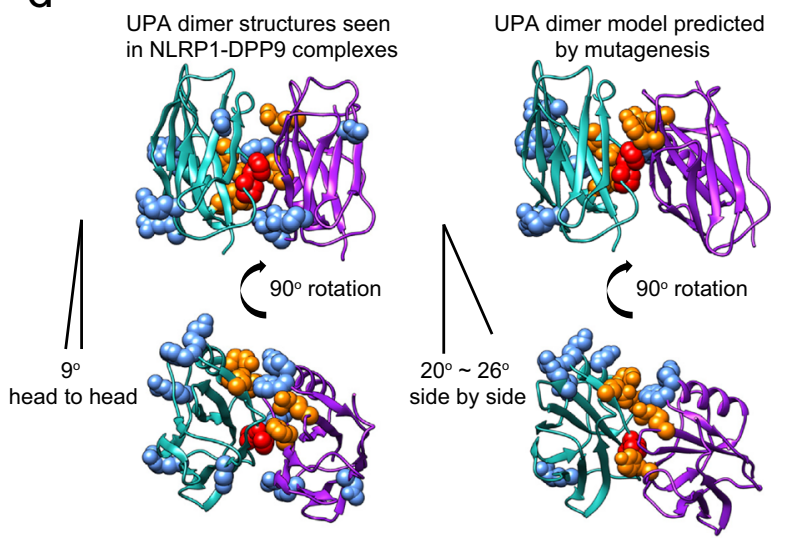

Modelled Linker Length

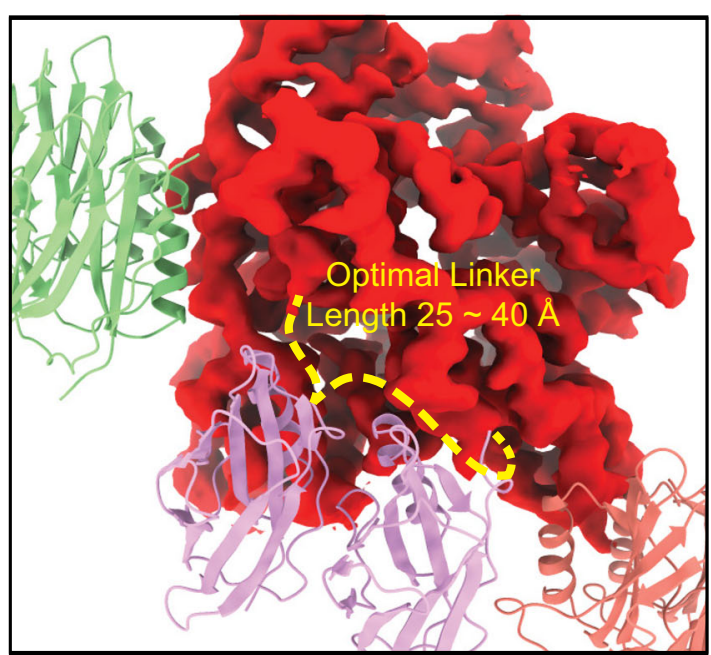

which had not been highlighted by previous analyses $24,29,34-40$. In ASC-CARD filaments, this bridge consists of two residues Gln185 and Tyr187, which extend from the Type IIb surface and interact with Ala168 and Asn170 on the Type IIa surface (Fig. 5a). In CASP1-CARD filaments, the Type II interface bridge employs a distinct IIa surface composed of Lys64 and Gln67, which interacts with Type IIb residues, Asp80 and Tyr82 (Fig. 5b). This "bridgelike" feature across the Type II interface becomes even more apparent when we refined the existing CASP1-CARD model (Supplementary Fig. 6c). Hence, a strong inter-subunit Type II density bridge seems to be a common feature amongst inflammasome CARD complexes. 
Fig. 3 Model of two-layer organization of NLRP1 and CARD8 FIINDUPA-CARD filaments. a Top panel: SDS-PAGE NLRP1-FIINDUPA-CARD mutants. Bottom panel: native gel analysis of SUMO-NLRP1-FIINDUPA constructs, stained by Krypton. b ASC-GFP speck formation induced by wild-type NLRP1FIINDUPA-CARD and PP1278AG in 293T-ASC-GFP cells. c Percentage of ASC-GFP specks induced by NLRP1-FIINDUPA-CARD constructs in 293T-ASCGFP cells. Cells were fixed 24 hours after transfection for fluorescence microscopy. Data are presented as mean values \pm SD. $P$ value was calculated with one-way ANOVA, $n=3$ biological replicates. “* ${ }^{\star \star \star} /{ }^{\star \star \star \star \prime}$ indicates $P$ value $<0.05,0.01$, and 0.0001 , correspondingly. $\mathbf{d}$ Side view and top view of FIIND UPA dimer structures reported by other groups, and our modeled oligomer interface of NLRP1-FIINDUPA. Potential surface residues that may involve homotypic oligomerization were displayed using spheres, PP1278-1279, were highlighted in red color, R1240, RK1260, R12785, and F1320 are colored in orange. e The top and side views of NLRP1-CARD filament and six modeled FIINDUPA domains fitted into the FIINDUPA-CARD filament density. $\mathbf{f}$ A close-up view of the potential UPA-CARD linker orientation. A linker with $25 \sim 40 \AA$ is likely to support a regular organization of the two-layer structure. $\mathbf{g}$ The top and side views of CARD8-CARD filament and si modeled FIINDUPA domains fitted into the CARD8-FIINDUPA-CARD filament density.

Using the refined assignment of Type II interface residues, we modeled all the pairwise unidirectional CARD-CARD interactions among NLRP1, CARD8, ASC, and CASP1 (Fig. 5c-f), based on our in-house built filament models (PDB-6K7V, 6K9F, 6K99) and newly refined CASP1-CARD oligomer structure (locally refined PDB-5FNA using our CARD8-CARD structure as a reference). A remarkable level of specificity becomes apparent, as certain interactions are favored, whereas others show strong incompatibility. NLRP1-CARD and ASC-CARD are expected to be compatible, as the prominent HPH (a.a. 1452-1454) motif at the NLRP1 Type IIb interface can form ionic interactions with the ASC IIa residues AWN (a.a. 168-170) (Fig. 5c, d, left panel). In contrast, the same NLRP1 HPH motif clashes with the CASP1 Type IIa surface residues, KGAQ (a.a. 64-67) (Fig. 5c, d, right panel, circle). In other words, NLRP1-CARD uses the HPH motif on its Type IIb surface to preferentially engage ASC-CARD and disfavor CASP1-CARD (Fig. 5c, d). On the other hand, CARD8-CARD employs a DPY (a.a. 525-527) motif on its Type IIb surface. This allows CARD8-CARD to interact strongly with the CASP1-CARD Type IIa surface via ionic interactions between Asp 525 (CARD8) and Lys64 (CASP1) (Fig. 5c, f, right panel). In contrast, the same DPY motif lacks effective interaction with the ASC-type IIa surface, as the charged Asp 525 in the CARD8 DPY motif cannot pair with Ala 168 on the ASC-type IIa surface (Fig. 5f, left panel). Hence, CARD8-CARD is structurally compatible with CASP1-CARD but not ASC-CARD. These results demonstrate that NLRP1-CARD and CARD8-CARD have the intrinsic ability to discriminate between ASC and procaspase- 1 due to the structural features of their Type IIb motifs.

This analysis can be further extended to explain why ASC is uniquely suited to act as an adaptor and amplifier in inflammasome signaling. To do so, ASC-CARD must engage upstream sensors, self-oligomerize, and activate CASP1-CARD simultaneously. This versatility is endowed by its unique Type IIb motif, QSY (a.a. 185-187), which possesses biochemical properties and charges that are intermediate between NLRP1-CARD and CARD8-CARD. The Type IIb QSY motif in ASC-CARD adopts a conformation that is compatible both with its own Type IIa residues, i.e., a.a. AWN (a.a. 168-170), as well as the CASP1 "KGAQ" motif (Fig. 5e). This is achieved with the help of additional sidechain features. For instance, instead of a prominent negative Asp residue in CARD8 Type IIb surface, ASC-CARD uses a less-charged Asn residue to enhance the interaction with Ala168 on its own type IIa surface. In addition, the tyrosine residue (Tyr187) on ASC-CARD Type IIb demonstrate a more inwards orientation as compared to the equivalent tyrosine residue on CARD8 type IIb surface (Tyr527), thus allowing it to effectively engage both its own Type IIa as well as that of CASP1CARD (Fig. 5e).

To further corroborate that signaling specificity is intrinsic to the CARD domains, we performed an in vitro oligomerization assay using recombinant fluorescently labeled CASP1-CARD and
ASC-CARD. In agreement with the structural predictions, CARD8-CARD, but not NLRP1-CARD, preferentially stimulated CASP1-CARD oligomerization, as demonstrated by the appearance of slower migrating bands on native gels (Supplementary Fig. 6f, left panel, lanes 2 and 3 vs. 4 and 5), whereas the reverse was true for ASC-CARD oligomerization (Supplementary Fig. 6f, right panel, lanes 2 and 3 vs. lanes 4 and 5). Next, we used structured illumination microscopy (3D-SIM) to directly visualize NLRP1-CARD and CARD8-CARD and their interactions with downstream CARDs (ASC-CARD vs. CASP1-CARD) within cultured human cells. Both mCherry-tagged NLRP1-CARD and CARD8-CARD formed filament bundles within the cytosol (Fig. 6a, red), which intertwined with those formed by GFPtagged ASC-CARD and CASP1-CARD, respectively (Fig. 6a, green). Given the theoretical resolution of $\sim 110 \mathrm{~nm}$ in width $(x-y$ plane), we postulated that the thinnest filament bundle observed was no more than approximately six times the width of an individual filament with the mCherry tag. In many instances, ASC-CARD or CASP1-CARD filaments could be observed as "emanating: from one side of NLRP1-CARD or CARD8-CARD filament bundles (Fig. 6a). This "filament-seeding" phenomenon was not limited to the isolated CARDs, as Talabostat-activated full-length NLRP1 and CARD8 also formed filaments ${ }^{9,15,22}$ and colocalized with ASC-CARD and CASP1-CARD filaments, respectively (Supplementary Fig. 7). Furthermore, Talabostatactivated full-length NLRP1, or its activating fragment NLRP1FIIND ${ }^{\text {UPA_CARD }}$ are unable to trigger CASP1-GFP filaments in the absence of ASC, whereas CARD8 (or CARD8-FIINDUPA_ CARD) was unable to nucleate ASC-GFP specks in $293 \mathrm{~T}$ cells (Supplementary Fig. 8a). Taken together, these results provide direct cellular evidence that NLRP1-CARD and CARD8-CARD form filament-like oligomers that differentially engage either ASC or pro-caspase-1, via heterotypic CARD interactions.

We further corroborated that CARD8 is the first human inflammasome sensor that is unable to engage ASC as an amplifier ${ }^{18}$. In immortalized human keratinocytes, CRISPR/Cas9mediated deletion of endogenous ASC (PYCARD) abrogated the ability of overexpressed NLRP1-FIINDUPA_CARD fragment or NLRP1-CARD to induce endogenous IL- $1 \beta$ secretion (Fig. $6 c$, red bars, Supplementary Fig. 8c-e) and pyroptotic cell death (Fig. 6d, red bars, Supplementary Fig. 8b, d). In contrast, CARD8dependent pyroptosis was not abrogated in ASC (PYCARD) KO keratinocytes (Fig. 6c, d, blue bars, Supplementary Fig. 8c, e). Thus, NLRP1 requires endogenous ASC for inflammasome activation in human cells, whereas CARD8 can directly activate caspase- 1 independently of ASC.

Finally, we performed a classical "seeding" experiment where purified untagged NLRP1-CARD or CARD8-CARD (the seed) was incubated with the respective SNAP-tagged partner CARD, i.e., ASC-CARD or CASP1-CARD. In this setup, the downstream ASC-CARD or CASP1-CARD filaments appear thicker due to the SNAP tag, and could therefore easily be distinguished from the upstream filaments composed of 
a
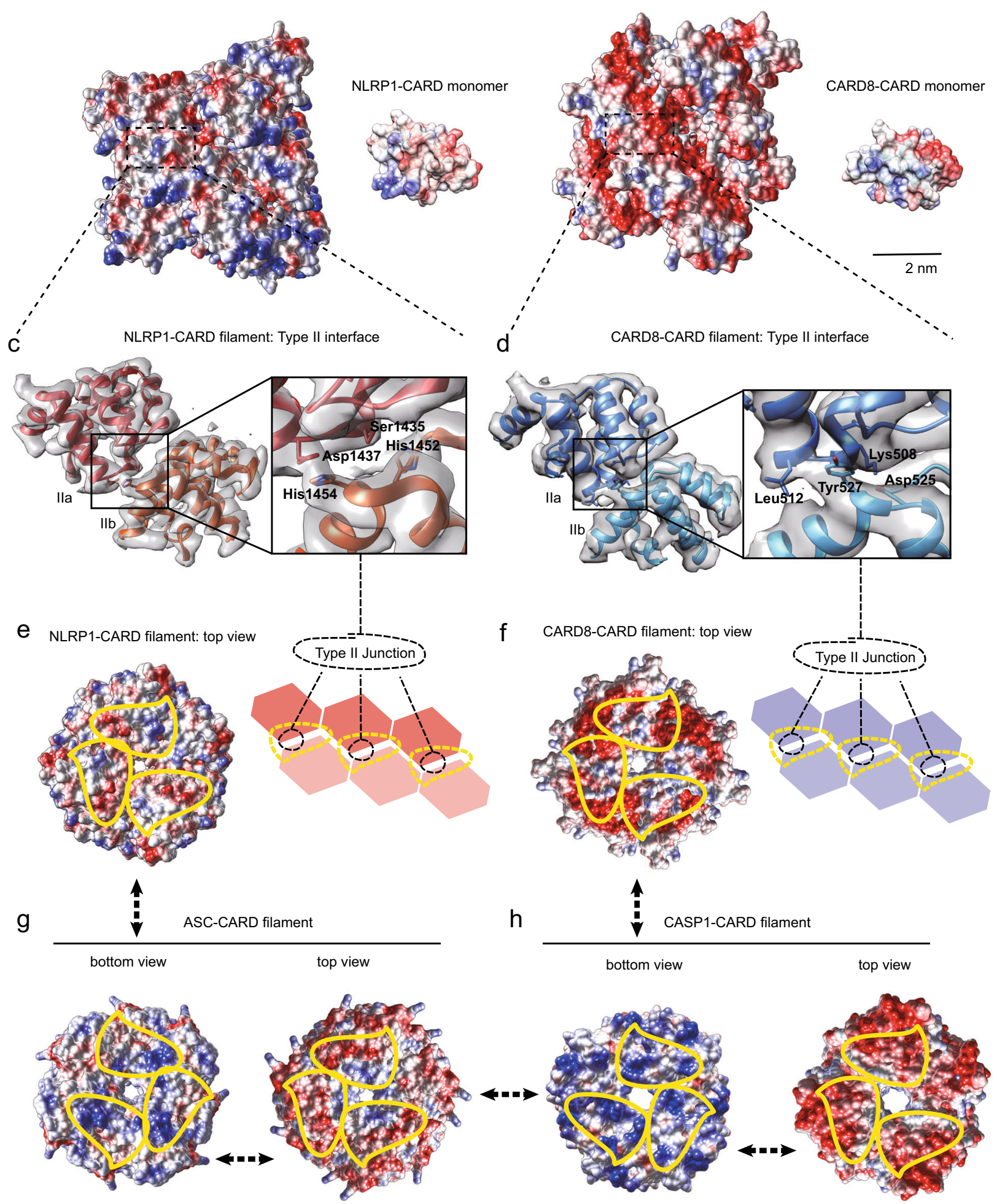

NLRP1- or CARD8-CARD. In several independent experiments, the thick downstream CARD (ASC-CARD and CASP1CARD) filaments could be seen to extend from one end of the thin NLRP1 and CARD8 upstream seeding filaments (Supplementary Fig. 9a, b). This is consistent with a structural model where oligomerized NLRP1-CARD and CARD8-CARD use a single type II interface to seed ASC-CARD or CASP1-CARD in a unidirectional manner.

NLRC4-CARD can engage either ASC-CARD or CASP1CARD. Our results thus far demonstrate that subtle differences in the inflammasome CARD domain structures, particularly at the type II junctions, can directly dictate the signaling specificity of the NLR sensor proteins. To extend these results beyond NLRP1 and CARD8 and test the predictive power of our model, we examined a third CARD-containing NLR sensor, NLRC4. To unambiguously assign the Type II interface residues of human 
Fig. 4 Oligomeric interfaces on inflammasome CARD domains. a Left panel: side view of electrostatic potential distribution of a segment of NLRP1-CARD filament (red, $-10 \mathrm{C} / \mathrm{m}^{2}$; blue, $10 \mathrm{C} / \mathrm{m}^{2}$; generated in Chimera). Right panel: electrostatic potential map of a NLRP1-CARD monomer in the same orientation as the filamentous oligomer. $\mathbf{b}$ Left panel: side view of electrostatic potential distribution of a segment of CARD8-CARD filament ( $\mathrm{red},-10 \mathrm{C} / \mathrm{m}^{2} ; \mathrm{blue}, 10 \mathrm{C} / \mathrm{m}^{2}$ ). Right panel: electrostatic potential map of a CARD8-CARD monomer in the same orientation. c Atomic model cartoon and the directly observed density map of a NLRP1-CARD dimer within the filamentous complex, centered at the Type II junction interface. The four residues, His1452-His1454 on the Type IIb interface and the Ser1435-Asp1437 on the Type Ila interface were highlighted and shown in the "stick" mode. d Atomic model cartoon and the directly observed density map of CARD8-CARD dimer in the filament, centered at the Type II junction interface. The four residues, Asp525-Tyr527 on the Type Ilb interface and the Lys508-Leu512 on the Type Ila interface were highlighted and shown in the "stick" mode. e Electrostatic potential map of the top view (Type Ilb interface, highlighted in yellow dashed area) of the NLRP1-CARD filament. $\mathbf{f}$ Electrostatic potential map of the top view (type Ilb interface, highlighted in yellow dashed area) of CARD8-CARD filament. $\mathbf{g}$ Electrostatic potential map of the bottom (type lla highlighted in yellow dashed area) and top (type Ilb highlighted in yellow dashed area) views of the ASC-CARD filament. $\mathbf{h}$ Electrostatic potential map of the bottom (type lla highlighted in yellow dashed area) and top (type Ilb highlighted in yellow dashed area) views of the CASP1-CARD filament.

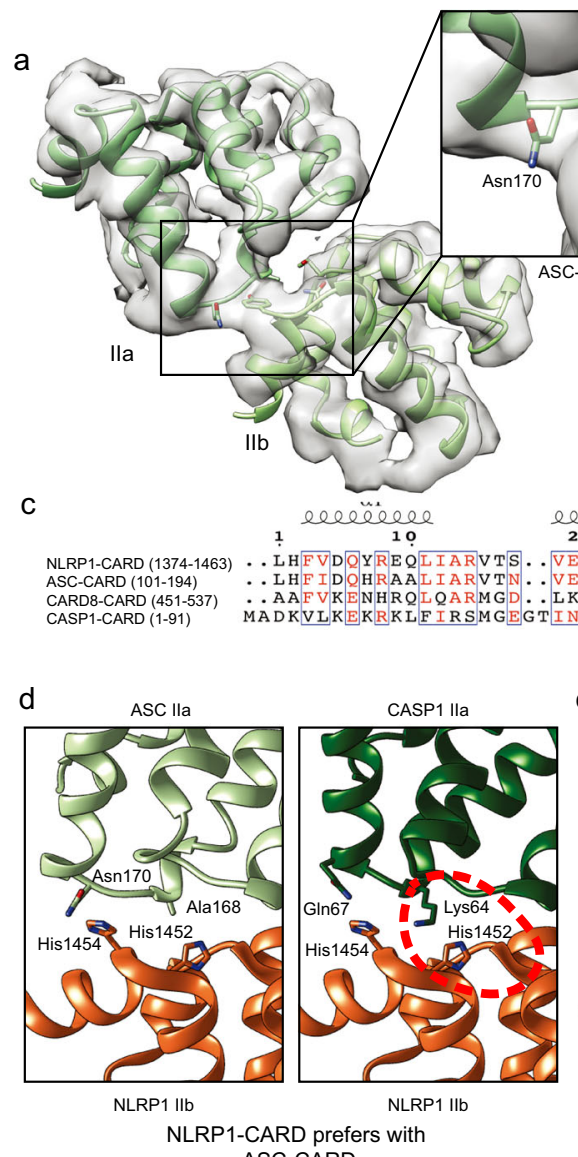

ASC-CARD

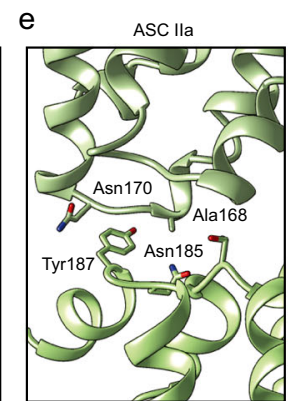

ASC IIb

ASC-CARD is compatible with both ASC-CARD and CASP1-CARD

ASC IIb

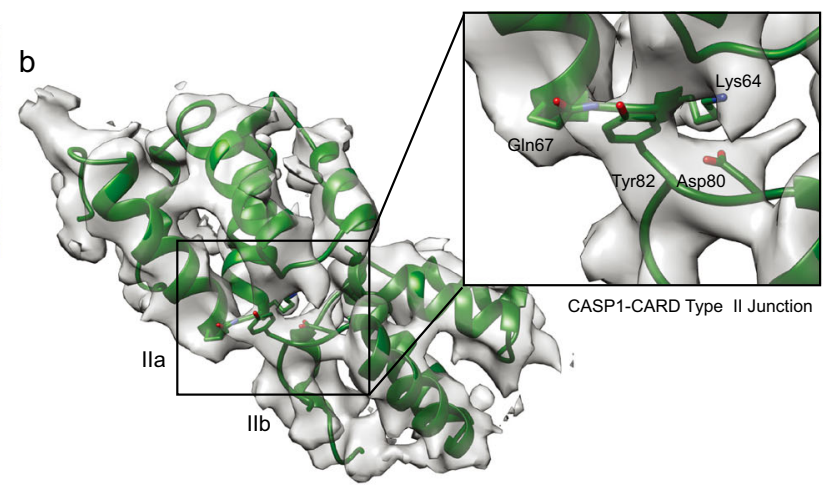

d2 20

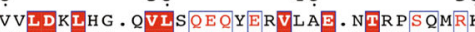
KLFSLSOSWDFCK TG

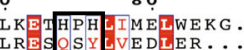
LVEDLER . .

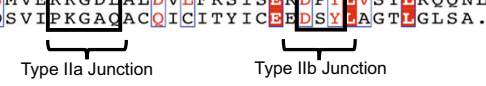
$\mathrm{f} \quad$ ASC lla
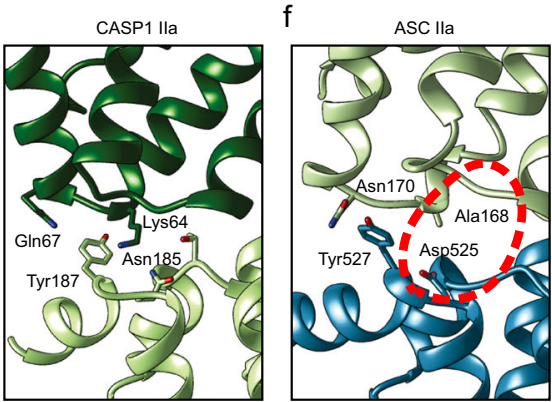

CARD8 IIb CARD8-CARD prefers CASP1-CARD

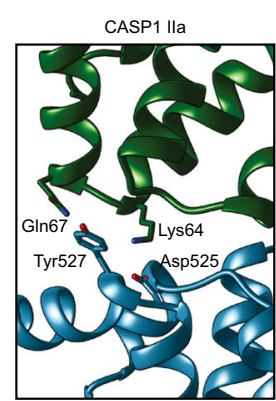

CARD8 IIb

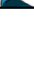

Fig. 5 Type Ila-b interface influences human inflammasome complexes. a Type II interface in ASC-CARD filaments (PDB-6K99, EMD-9947). b Type II interface in CASP1-CARD filaments after refitting. See Fig. S5c for a comparison between the refitted model and the original model, PDB-5FNA, and EMD3241). c Sequence alignment of human NLRP1-, ASC-, CARD8-, and CASP1-CARD. Important residues within the Type II interfaces are highlighted in black box. d Structural compatibility of human NLRP1-CARD with ASC-CARD or CASP1-CARD. Potential clashes are highlighted in red. e Structural compatibility of human ASC-CARD with itself or CASP1-CARD. $\mathbf{f}$ Structural compatibility of human CARD8-CARD with ASC-CARD or CASP1-CARD. Potential clashes are highlighted in red.

NLRC4-CARD, we determined its filament structure at $3.3 \AA$ using cryo-EM (Supplementary Fig. 6d, e). The final polished structure closely agrees with previously published findings ${ }^{24,39}$. Interestingly, unlike NLRP1-CARD and CARD8-CARD, the strong density bridge in the Type II interface is absent within NLRC4-CARD filaments (Supplementary Fig. 10a). The Type IIb tyrosine residue (Tyr78) is folded backward toward the hydrophobic core and does not participate in intermolecular Type II interaction. Instead, two nearby small residues, Asn77 and Gln82, come forward to form a class of Type II junction (Supplementary Fig. 10a, b) that is more flexible than that of NLRP1 and CARD8.
Molecular docking predicted that NLRC4 Type IIb surface would be structurally compatible with both ASC-CARD and CASP1CARD. This was validated using the in vitro EMSA assay (Supplementary Fig. 6f, lanes 6-7 in both panels) and in $293 \mathrm{~T}$ cells. Overexpressed NLRC4-CARD nucleated the formation of both ASC-GFP and CASP1 C285G-GFP specks, as well as ASCCARD-GFP and CASP1-CARD-GFP filaments at similar levels (Supplementary Fig. 10c, d). The versatility of human NLRC4CARD is in agreement with previous studies on murine Nlrc4. In mice, Asc/Pycard knockout attenuates, but does not abolish caspase-1-dependent pyroptotic cell death ${ }^{41}$, suggesting that 
a

b
3D-SIM reconstructed contour plot
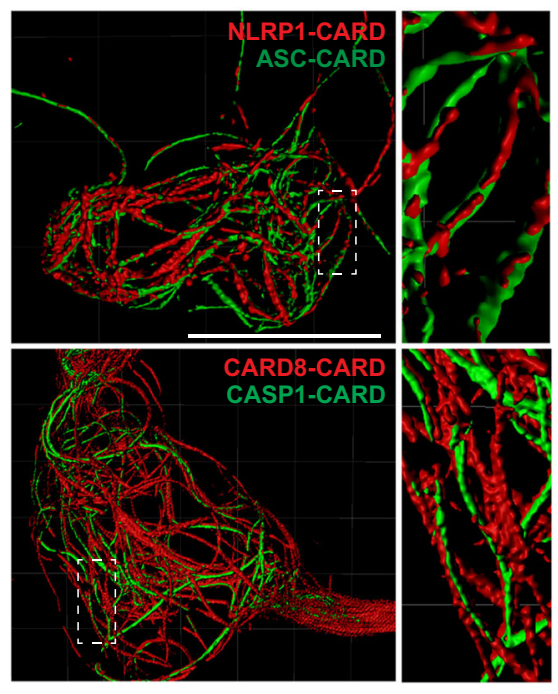

C

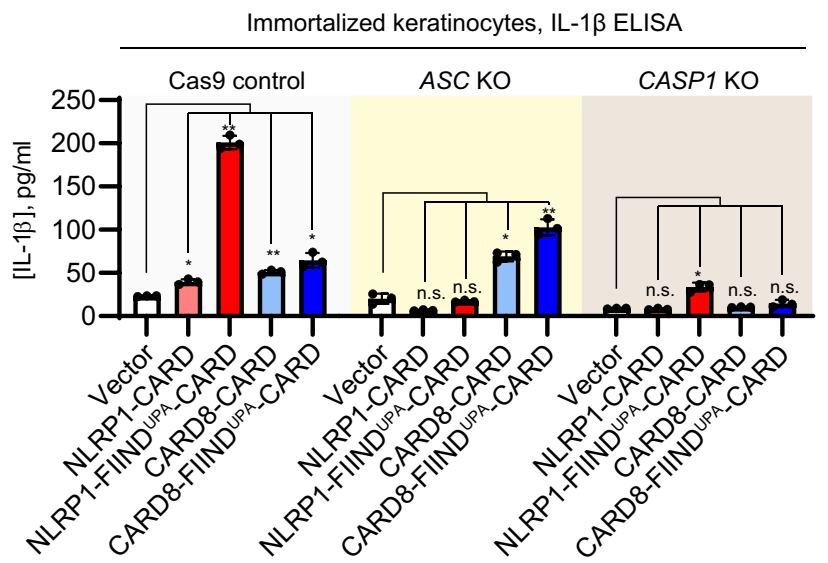

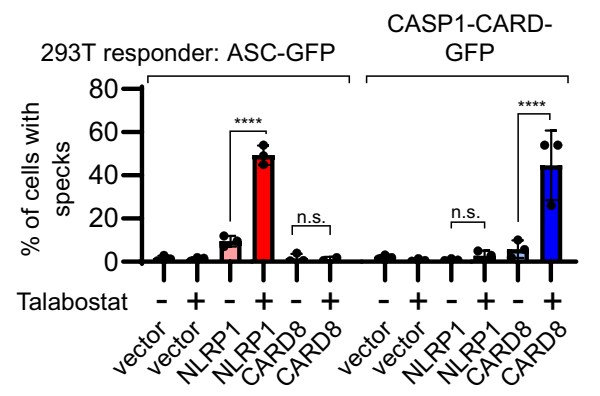

d

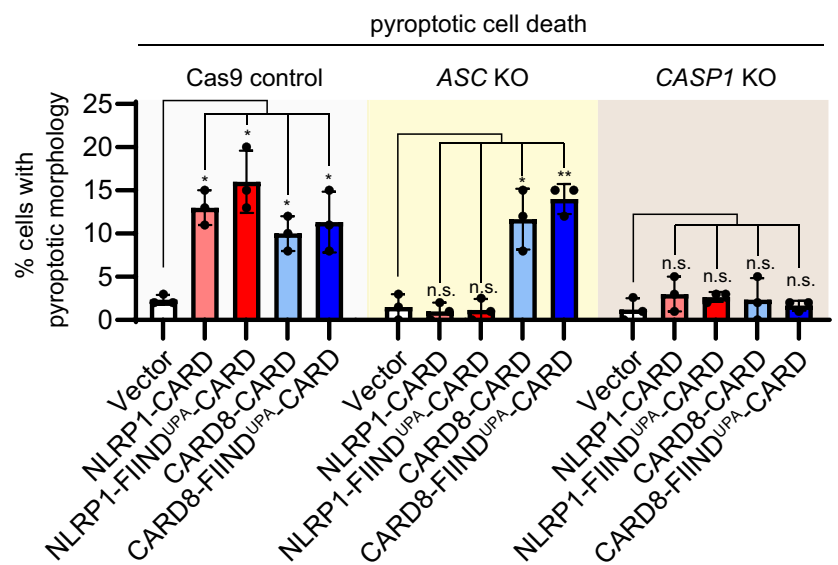

Fig. 6 NLRP1 and CARD8 inflammasome assembly in mammalian cells. a Structured illumination microscopy images of NLRP1-CARD-mCherry and CARD8-CARD-mCherry filaments (red) with ASC-CARD-GFP and CASP1-CARD-GFP filaments (green). White boxes indicate regions magnified in right panels. b Percentage of ASC-GFP specks or CASP1-CARD-GFP filaments induced by full-length NLRP1 or CARD8 upon Talabostat treatment (3 $\mu$ M). Data are presented as mean values \pm SD. $P$ value was calculated with one-way ANOVA, $n=3$ treatments. ${ }^{\star \star} /{ }^{\star \star} /{ }^{\star \star * \star \prime}$ indicates $P$ value $<0.05,0.01$, and 0.0001 , correspondingly. c IL-1 $\beta$ secretion in control, ASC KO, and CASP1 KO keratinocytes transfected with the indicated NLRP1 and CARD8 constructs. Data are presented as mean values \pm SD. $P$ value was calculated with one-way ANOVA, $n=3$ transfections. ${ }^{\star \star} /{ }^{\star \star} /{ }^{\star \star \star \star \star \prime}$ indicates $P$ value $<0.05,0.01$, and 0.0001 , correspondingly. d The percentage of cells with pyroptotic morphology in control, ASC KO, and CASP1 KO keratinocytes transfected with the indicated NLRP1 and CARD 8 constructs. Data are presented as mean values \pm SD. $P$ value was calculated with one-way ANOVA, $n=3$ transfections (same as $5 \mathrm{c}$ ).

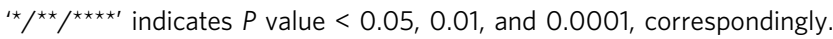

murine NLRC4, similar to its human homolog can engage both ASC and pro-caspase-1 (Supplementary Fig. 10e).

\section{Discussion}

Taken together, our cellular, biochemical, and structural findings suggest a model for NLRP1- and CARD8 inflammasome activation (Fig. 7). We propose that FIIND auto-cleavage, which has been shown previously to be required for NLRP1 activation, serves as a necessary "licensing" step to liberate the FIIND UPA domain. Upon ligand-induced or $3 \mathrm{C}$ protease-mediated ${ }^{42}$ activation and relief of auto-inhibition, FIIND ${ }^{\text {UPA }}$ self-assembles into ring-like oligomers in a "head-to-tail" fashion (Fig. 3), bringing adjacent CARD monomers into close proximity. This lowers the threshold CARD oligomerization and allows for further "prionoid"-like growth of FIIND UPA_CARD filaments (Fig. 7a).

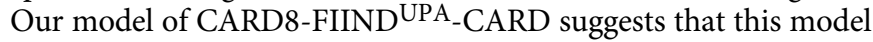
likely also holds true for the CARD8 inflammasome (Fig. 7b).

We next solved the cryo-EM structures of NLRP1-CARD and CARD8-CARD filaments. Despite very similar helical parameters, the subtle differences in rotational angles of individual NLRP1CARD and CARD8-CARD monomers and their type II junctions give rise to distinct surface charge distributions on the filament scale. Extending this analysis, we also found that specific type II interface motifs directly dictate the compatibility of heterotypic pairwise CARD-CARD interactions among NLRP1, CARD8, NLRC4, ASC, and CASP1. In particular, these results explain why NLRP1, but not CARD8, requires ASC for inflammasome activation. Thus, the CARD domains are not mere interchangeable "lego building blocks". Instead, each possesses intrinsic abilities to 
a

Human NLRP1

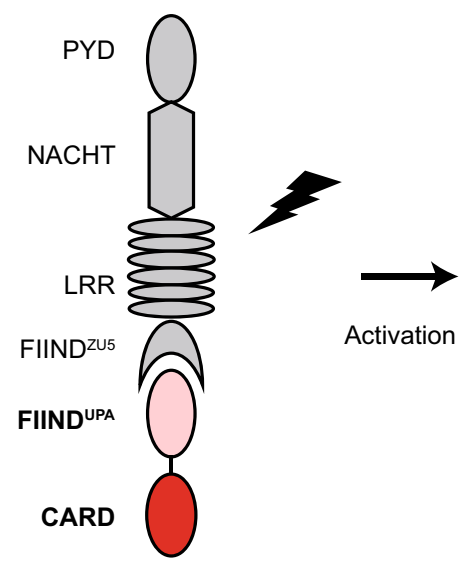

Specific Type II interaction with ASC-CARD

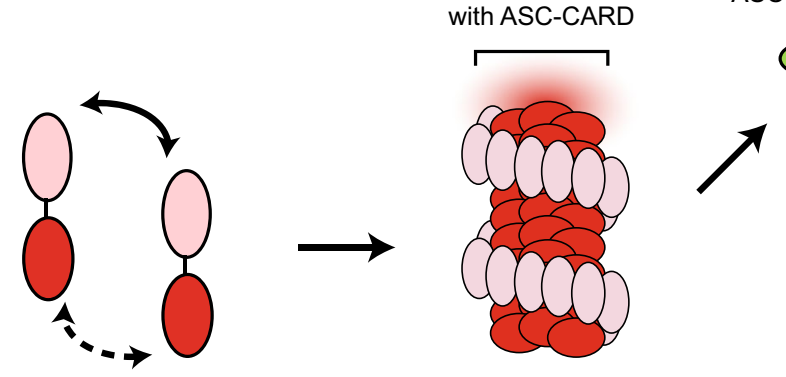

AS oligomerization

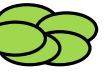

\section{FIIND ${ }^{\text {UPA }}$ facilitated}

CARD oligomerization

b

Human CARD8
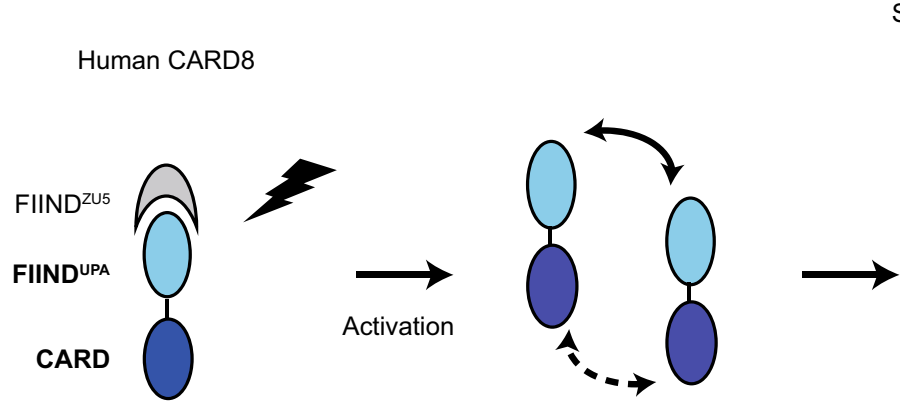
Specific Type II interaction
with CASP1-CARD
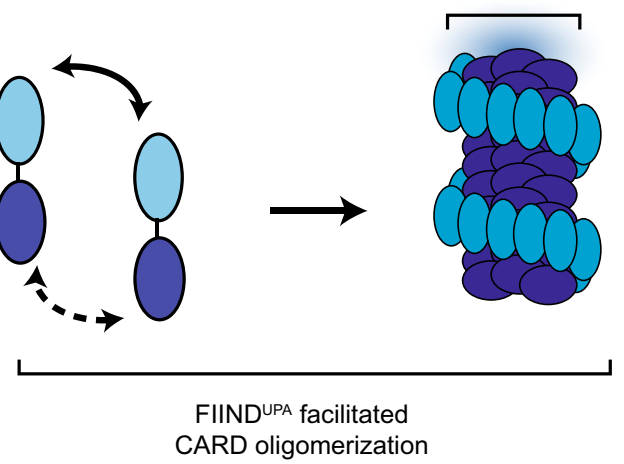

ASC
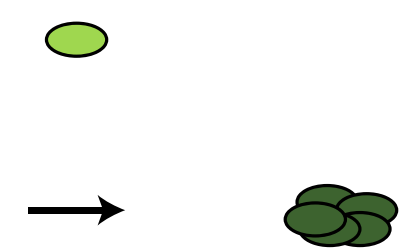

ASC-independent CASP1 activation

CARD oligomerization

Fig. 7 A two-step model for human NLRP1 and CARD8 inflammasome assembly. From left to right, NLRP1 (a) and CARD8 (b) are "licensed" by autoproteolysis within the FIIND. Upon activation by PAMP or DAMP triggers, the self-inhibition exerted by the $\mathrm{N}$-terminal proteolytic fragment is relieved. FIINDUPA facilitates the oligomerization of CARD, leading to the formation of a two-layered filament complex. Oligomerized NLRP1-CARD and CARD8CARD differentially engage ASC or CASP1, leading to the assembly of distinct inflammasome complexes.

discriminate amongst potential downstream binding partners in order to specify the composition of different inflammasome complexes. It is conceivable that these distinct inflammasome complexes can lead to distinct biological outcomes, in agreement with previous findings demonstrating that different strengths of the pyroptotic signaling are required for cell death and IL-1 cytokine secretion ${ }^{41,43}$. Interestingly, the prominent role in type II interface-dependent assembly is apparently specific to the inflammasome complexes, as other CARD domain innate immune complexes, such as MAVS (EMD-5925) ${ }^{33}$, BCL10 $\left(\right.$ EMD-7314) 44 , RIPK2 (EMD-4399, 6842) ${ }^{35,45}$ rely more heavily on Type I interface junctions (e.g., MAVS Arg43-Typ56 density bridge). This underscores the importance of studying molecular structures of large complexes at atomic resolutions. As supramolecular assemblies are highly prevalent in innate immune and cell death signaling ${ }^{46}$, our findings should inform future work to decipher the general assembly principles of other molecular complexes involved in these vital processes.

\section{Methods}

Cell culture. HEK293Ts (ATCC \#CRL-3216) were obtained from commercial sources and cultured according to the suppliers' protocols. Immortalized human keratinocytes (N/TERT-1) were a kind gift from H. Reinwald (MTA ${ }^{47}$. All cell lines underwent routine Mycoplasma testing with Lonza MycoAlert (Lonza \#LT07-118).

Transient transfection and stable cell line generation using lentiviruses. 293TASC-GFP cells were described previously ${ }^{8}$. All other 293T-GFP responder cell lines were constructed by lentiviral transduction ( $\mathrm{pCDH}$ vector backbone, System Biosciences). All transient expression plasmids were cloned into the $\mathrm{pCS} 2+$ vector using standard restriction cloning. Polyclonal Cas9/CRISPR knockout cell lines were generated with lentiCRISPR-v2 (Addgene \#52961) and selected with puromycin. Knockout efficiency was tested with western blot 7-10 days after puromycin selection. Site-directed mutagenesis was carried out with QuickChangeXI II (Agilent \#200522).

3D-SIM and widefield-deconvolution. A DeltaVision OMX v4 Blaze microscope (GE Healthcare) equipped with $488 \mathrm{~nm}$ and $568 \mathrm{~nm}$ lasers for excitation (3D-SIM) and solid-state illuminator (widefield) the BGR-FR filter drawer (emission wavelengths $436 / 31$ for DAPI) was used for acquisition of 3D-SIM and widefielddeconvolution images. An Olympus Plan Apochromat $\times 100 / 1.4$ point spread function oil immersion objective lens was used with liquid-cooled Photometrics Evolve EM-CCD cameras for each channel. For 3D-SIM, 15 images per section per channel were acquired (made up of three rotations and five-phase movements of the diffraction grating) at a $\mathrm{z}$-spacing of $0.125 \mu \mathrm{m}$. The same $\mathrm{z}$-spacing was used for widefield acquisition. Structured illumination reconstruction or deconvolution and alignment was completed using the SoftWorX (GE Healthcare) program, 3D image rendering and analysis in Imaris version 9.3.0 (Bitplane an Oxford Instruments 
Company), 2D image analysis in Fiji (Schindelin et al., 2012) and figure preparation in Illustrator.

Antibodies and cytokine analysis. The following antibodies were used in this study: HA tag (Santa Cruz Biotechnology, \#sc-805, 1:1000 for western blot), GAPDH (Santa Cruz Biotechnology, \#sc-47724, 1:1000 for western blot), ASC (Adipogen, \#AL-177, 1:1,000 for western blot), CASP1 (Santa Cruz Biotechnology, \#sc-622, 1:1000 for western blot), IL-1B (R\&D systems, \#AF-201, $0.1 \mu \mathrm{g} / \mathrm{mL}$ for western blot, $2 \mu \mathrm{g} / \mathrm{mL}$ for immunochemistry), FLAG (SigmaAldrich, \#F3165, 1:2000 for western blot), GFP (Abcam, \#ab290, 1:2,000 for western blot). IL-1B measurements were carried out with human IL-1B ELISA kit (BD, \#557953, follow supplier instructions).

Plasmid construction for recombinant protein expression. For recombinant CARD proteins, NLRP1 constructs (985-1473, 1213-1473, 1374-1466, 1374-1473) with or without C-terminal SNAP tag (NEB), CARD8-CARD (451-537) with or without C-terminal SNAP tag, ASC-CARD (1-102) with N-terminal SNAP tag, CASP1-CARD (1-92) with or without C-terminal SNAP tag, NLRC4-CARD (1-102) with or without C-terminal SNAP tag. were cloned into pET47 vector in between XmaI and XhoI restriction sites. A 3 C protease cleavage site was inserted in between the CARD domain and the SNAP tags in case SNAP needs to be removed for biochemical analysis. Site-directed mutagenesis was performed using the KAPA Hifi PCR kits (KAPA Biosystems).

\section{Protein expression and purification. For the expression of the His-tagged} recombinant proteins, the constructed plasmids were transformed into BL21(DE3) (NEB) and grown at $37^{\circ} \mathrm{C}$ for $16-18 \mathrm{~h}$. Then the starting culture was transferred to a larger volume of lysogeny broth, which was grown until an OD600 of $\sim 0.6-0.8$, then the temperature was lowered to $16^{\circ} \mathrm{C}$, and the cells were induced with $0.5 \mathrm{mM}$ IPTG and grown overnight. Bacteria were harvested at $4600 \times g$ (Avanti JXN series, Beckman Coulter) for $10 \mathrm{~min}$ and then resuspended with lysis buffer (20 mM Tris$\mathrm{HCl}, \mathrm{pH} 8.0,300 \mathrm{mM} \mathrm{NaCl}, 20 \mathrm{mM}$ imidazole, and $10 \%$ glycerol). Cells were then lysed by Emulsiflex-C3 and centrifuged at a speed of $30,000 \times g$ (Avanti JXN series, Beckman Coulter) for $20 \mathrm{~min}$ at $4^{\circ} \mathrm{C}$ to separate supernatant and pellet. The supernatant was passed through a pre-equilibrated column containing Ni-NTA agarose beads (ThermoFisher Scientific) by gravity. The column was then washed with lysis buffer containing $20 \mathrm{mM}$ imidazole to remove nonspecific binding proteins. His-tagged proteins were eluted with elution buffer that contained $300 \mathrm{mM}$ imidazole. To further purify our recombinant proteins, size-exclusion chromatography (Superdex 200 increase, GE Healthcare) was performed in buffer A $(20 \mathrm{mM}$ Tris- $\mathrm{HCl}, \mathrm{pH} 8.0,150 \mathrm{mM} \mathrm{NaCl}$, and $1 \mathrm{mM} \mathrm{DTT}$ ). At this step, the purified proteins were the oligomeric pre-activated seed fraction. For monomeric CARD-SNAP or SNAP-CARD domain proteins, it was prepared following the protocol as described here. Concentrated and purified soluble filament seed at around $10 \mathrm{mg} / \mathrm{ml}$ was buffer exchanged into $10 \mathrm{ml}$ of $6 \mathrm{M}$ guanidinium, and gradually dialyzed against $3,2,1.5,1,0.8$, and $0.6 \mathrm{M}$ guanidinium containing buffer $\mathrm{A}$ in the cold room, and eventually in buffer A. In the absence of stimuli, refolded SNAP-ASC-CARD protein remained monomeric for $24 \mathrm{~h}$, and CASP1-CARD-SNAP remained monomeric or lower oligomeric for about $12 \mathrm{~h}$. Recombinant SNAP-NLRP1-FIIND UPA_CARD and SNAP-CARD8-FIINDUPA-CARD were expressed in similar ways as the CARD domain constructs. Notably, most of the protein exist in cellular pellets after the Emulsiflex step, thus, direct pellet wash with lysis buffer with an additional $1 \%$ Triton-X100, followed by $6 \mathrm{M}$ Guanidinium is necessary to obtain solution proteins. Chemical refolding following the same dialysis protocol, followed by $3^{\circ} \mathrm{C}$ cleavage of the SNAP tag, was used to obtain homogenous oligomeric population of FIIN$\mathrm{D}^{\mathrm{UPA}}$-CARD complexes at various concentrations.

In vitro oligomerization assay. SNAP-ASC-CARD and CASP1-CARD-SNAP monomers that were labeled with Alexa Fluor 647 (New England Biolabs) were coincubated with CARD domain only filament seeds (CARD8-CARD without SNAP, NLRP1-CARD without SNAP, NLRC4-CARD without SNAP) separately. One microliter of $100 \mu \mathrm{M}$ BG-Alexa dye stocks (dissolved in water containing 5\% dimethyl sulfoxide) were added into every $100 \mu \mathrm{l}$ of pre-labeling protein stock at a concentration of $20 \mu \mathrm{M}$. Fluorescently labeled monomeric SNAP-ASC-CARD $(10 \mu \mathrm{M})$ and CASP1-CARD-SNAP $(10 \mu \mathrm{M})$ was mixed with 20 or $5 \mu \mathrm{M}$ seed oligomer for $15 \mathrm{~min}$ at room temperature before analysis by Bis-Tris native PAGE gel (Life) or $15 \%$ sodium dodecyl sulfate polyacrylamide gel electrophoresis gel. The fluorescently labeled gel was run at a voltage of $200 \mathrm{~V}$ for $60 \mathrm{~min}$. Once the EMSA was completed, they were visualized by Typhoon FLA7000 (GE Healthcare) with $100-\mu \mathrm{m}$ pixel size at channel 647 using PTM 600. To form ASC-CARD and NLRC4-CARD long filament $(200-500 \mathrm{~nm}), \sim 20 \mu \mathrm{M}$ monomeric SNAP-tagged protein was mixed with unlabeled, wild-type SNAP-tagged ASC-CARD and NLRC4-CARD seeds at a molar ratio of 20:1 for overnight. Later on, in-houseprepared MBP-3C-protease was added to the fully extended filament with a final concentration of $0.5 \mu \mathrm{M}$. The digestion was performed at $4{ }^{\circ} \mathrm{C}$ for $8 \mathrm{~h}$, before further size-exclusion purification to separate the core filament, the cleaved-off SNAP tags, and proteases. Final extended ASC-CARD and NLRC4-CARD thin filament were then purified using size-exclusion column Superose 6 (GE Healthcare), before structural analysis. To form long NLRP1-CARD and
CARD8-CARD filaments, $\mathrm{N}$-terminal His-tagged CARD domain alone constructs were expressed and purified according to the protocol mentioned above, then concentrated to $\sim 400 \mu \mathrm{M}$, homogeneous long filaments formed spontaneously, without the requirement of seeding.

Negative stain EM. Samples were applied onto the grids and stained following a Harvard medical school Liao lab protocol (https://liao.hms.harvard.edu/node/32, originally designed by Tom Walz). Protein samples were diluted to $0.01-0.05 \mathrm{mg} /$ $\mathrm{ml}$ for best sample density on the grids, immediately before grid preparation to prevent concentration-dependent dissociation. Images were taken using a T12 $120 \mathrm{keV}$ TEM microscope, at either $30,000 \times$ or $48,000 \times$ magnification, and calibrated against known objects of MAVS-CARD filament.

NLRP1-FIIND ${ }^{\text {UPA }}$-CARD complex density model was built based on 58 negative stained EM images with homogenous background, total 818 segments from a single $15 \mathrm{~nm}$ thick $2 \mathrm{D}$ class. Combining more segments yielded very noisy maps. Several preliminary maps $\sim 29 \AA$ were obtained after refinement, when different helical parameters were adopted, but all maps have the similar two-layer features. In addition, such strong inner layer density and spotted outer layer density are always seen in all solutions. The illustrated 3D map was obtained when choosing helical parameters similar to NLRC4 density EMDB-2901 and analysis of the NLRP1-FIIND ${ }^{\text {UPA }}$-CARD 2D class images. We were cautious about the accuracy of this particular map, thus simply using it as a guide to piece together an overall architecture of NLRP1 inflammasome.

Cryo-EM and helical reconstruction. For cryo-EM grid preparation, Quantifoil R1.2/1.3 holey grid (Quantifoil) was glow-discharged for $60 \mathrm{~s}$ and plunge-frozen using a Vitrobot (FEI) to be treated with $5-\mu \mathrm{l}$ sample at a concentration of $\sim 2 \mathrm{mg} /$ ml. Final high-resolution images were collected at Titan Krios, $300 \mathrm{kV}$, using Gatan K2 camera (Gatan), super-resolution mode for data collection, and binned $2 \times$ when doing motion correction. The sub-frame time is $250 \mathrm{~ms}$ and total exposure time is $10 \mathrm{~s}$, this will give 40 frames per stack. The pixel size on the final image is $1.10 \AA$. The dose rate is $4 \mathrm{e} / \mathrm{pixel} / \mathrm{s}$.

For cryo-EM data, super-resolution image stacks were "gain-normalized" and binned by $2 \times$ to a pixel size of $1.10 \AA$ prior to drift and local movement correction using MotionCorr2. Date processing software Relion 3.0 was used for CTF determination, particle picking, 2D classification, 3D classification, and refinement procedures. Totally, 2187 (NLRP1), 1424 (CARD8), 3183 (ASC), and 2109 (NLRC4) micrographs were collected and analyzed. We manually picked out $\sim 10,000$ to 20,000 filaments for each sample and windowed out segments of $240 \times 240$ pixels, yielding 422,388 (NLRP1), 1,201,108 (CARD8), 877,406 (ASC-CARD), and 382,715 (NLRC4) particles for 2D classification. After several iterations, bad particles and less desirable classes were removed and we used 100,240 (NLRP1), 301,848 (CARD8), 131,260 (ASC-CARD) and 120,602 (NLRC4) particles for 3D classification. $\mathrm{Ab}$ initio low-resolution helical structure was generated using a Gaussian cylinder as an initial model. 3D classification and auto-refinement of 53,241 (NLRP1), 98,280 (CARD8), 59,566 (ASC-CARD), and 120,602 (NLRC4) particles were done in RELION 3.0 and CisTEM. All 3D refinements were carried out in RELION. After refinement was converged, a mask of the central $30 \%$ segment was calculated and applied to the final data set and the corrected Fourier shell correlation (FSC) was calculated to estimate the resolution $\sim 3.7 \AA$ (NLRP1), $3.7 \AA$ (CARD8), $4.1 \AA$ (ASC-CARD), $3.3 \AA$ (NLRC4) by using FSC $=0.143$ criterion. In the final model, parameters of the RIP2 filament were calculated to yield $5.36 \AA$ (NLRP1), $5.409 \AA$ (CARD8), $5.27 \AA$ (ASC-CARD) and $5.17 \AA$ (NLRC4) of the axial rise per asymmetric unit and an azimuthal rotation per subunit of $-100,821^{\circ}$ (NLRP1), $-99,16^{\circ}$ (CARD8), $-100,614^{\circ}$ (ASC-CARD), $-100,55^{\circ}$ (NLRC4). Helical reconstruction parameters were determined using a real-space exhaustive parameter search method. About 1,600 combinations of helical parameters were applied to each data set and the output density was independently scored based on their biochemical properties. The simulated power spectrum of assigned helical parameters were also compared with the actual $2 \mathrm{D}$ images to confirm the accuracy of helical parameter search (Supplementary Fig. 11).

\section{Model building. Unpolished maps were used for model building.}

Step 1 was the monomer docking. The CARD monomer structures (PDB-4IFP for NLRP1-CARD, PDB-4KIM for CARD8-CARD, PDB-6N1H for ASC-CARD, and PDB-6N1I for NLRC4-CARD) were used as starting points at this stage. In the cases of NLRP1 and CARD8, the residue numbers were renumbered and the terminal residues were deleted to match the length of the density. The individual helix was manually shifted (rigid body movement) according to the locations of three large aromatic side chains. The density map at this resolution enabled us to unambiguously locate these residues. This step was done in Coot manually.

In Step 2, a tetramer containing the Type I, II, and III interfaces (a centrally located monomer and three other monomers forming Type I, II, and III interfaces with the central one) was built. After building four monomers in this way, we obtained a tetramer that could be used to refine the surface interactions. Phenix real-space refinement was done without rigid body refinement. Different parameters were tested, a torsion-rotational angle NCS constraints (Phenix default auto setting) was used at this step. In this way, all surfaces of the central monomer could be refined. 
During Step 3, a tetramer model was built that is in agreement with the data from the cellular activity assays, given particular emphasis in charge-reversal experiments.

In Step 4, a 12mer model was built. The central monomer from Step 3 was copy-and-pasted to occupy the entire $60 \AA$ segment density. In the end, 12 identical monomers were added to the density. Phenix real-space refinement with default NCS torsion-rotational constraint was performed. After several rounds of rigid body refinement, a model with reasonable statistics was obtained.

Step 5: Final refinement. Specific issues, like high clash score and Ramachandran plot outliers after Step 4 were manually identified and rebuilt and then Step 4 was repeated until satisfactory statistics and density interpretation were achieved.

ChimeraX was used to produce illustrations of atomic densities and molecular structure models.

Statistics and reproducibility. All negative stain EM experiments were reproduced at least two times using different batches of biological material. In particular, for material/constructs that were eventually used for structural analysis and determination (including NLRP1-FIIND ${ }^{\mathrm{UPA}}$-CARD, CARD8-FIIND ${ }^{\mathrm{UPA}}$-CARD, NLRP1-CARD, CARD8-CARD, ASC-CARD, NLRC4-CARD), at least four different batches of biological samples (independently expressed, purified, and seeded) were analyzed. All presented images were representative observations from these repeated experiments. Biochemical and cellular experiments were reproduced using at least three independent biological samples.

Reporting summary. Further information on research design is available in the Nature Research Reporting Summary linked to this article.

\section{Data availability}

The densities and structure models discussed in this study were validated and deposited in public databases. The accession codes and links to these files are: NLRC4-CARD filaments (PDB-6K8J, EMD-9946); ASC-CARD filaments (PDB-6K99, EMD-9947); NLRP1-CARD (PDB-6K7V, EMD-9943); CARD8-CARD (PDB-6K9F, EMD-9948). Other data are available from the corresponding authors upon reasonable request. Source data are provided with this paper.

Received: 3 April 2020; Accepted: 25 November 2020; Published online: 08 January 2021

\section{References}

1. Hou, F. et al. MAVS forms functional prion-like aggregates to activate and propagate antiviral innate immune response. Cell 146, 448-461 (2011).

2. Lin, S.-C., Lo, Y.-C. \& Wu, H. Helical assembly in the MyD88-IRAK4-IRAK2 complex in TLR/IL-1R signalling. Nature 465, 885-890 (2010).

3. Wen, H., Miao, E. A. \& Ting, J. P.-Y. Mechanisms of NOD-like receptorassociated inflammasome activation. Immunity 39, 432-441 (2013)

4. Lu, A. et al. Unified polymerization mechanism for the assembly of ASCdependent inflammasomes. Cell 156, 1193-1206 (2014).

5. Chen, G., Shaw, M. H., Kim, Y.-G. \& Nuñez, G. NOD-like receptors: role in innate immunity and inflammatory disease. Annu. Rev. Pathol. 4, 365-398 (2009).

6. Hoffman, H. M., Mueller, J. L., Broide, D. H., Wanderer, A. A. \& Kolodner, R. D. Mutation of a new gene encoding a putative pyrin-like protein causes familial cold autoinflammatory syndrome and Muckle-Wells syndrome. Nat. Genet. 29, 301-305 (2001)

7. Ogura, Y., Sutterwala, F. S. \& Flavell, R. A. The inflammasome: first line of the immune response to cell stress. Cell 126, 659-662 (2006).

8. Zhong, F. L. et al. Human DPP9 represses NLRP1 inflammasome and protects against autoinflammatory diseases via both peptidase activity and FIIND domain binding. J. Biol. Chem. 293, 18864-18878 (2018).

9. Zhong, F. L. et al. Germline NLRP1 mutations cause skin inflammatory and cancer susceptibility syndromes via inflammasome activation. Cell 167, 187-202.e17 (2016).

10. Johnson, D. C. et al. DPP8/DPP9 inhibitor-induced pyroptosis for treatment of acute myeloid leukemia. Nat. Med. 24, 1151-1156 (2018).

11. Finger, J. N. et al. Autolytic proteolysis within the function to find domain (FIIND) is required for NLRP1 inflammasome activity. J. Biol. Chem. 287, 25030-25037 (2012)

12. Grandemange, S. et al. A new autoinflammatory and autoimmune syndrome associated with NLRP1 mutations: NAIAD (NLRP1-associated autoinflammation with arthritis and dyskeratosis). Ann. Rheum. Dis. 76, 1191-1198 (2017)

13. Levandowski, C. B. et al. NLRP1 haplotypes associated with vitiligo and autoimmunity increase interleukin- $1 \beta$ processing via the NLRP1 inflammasome. Proc. Natl Acad. Sci. USA 110, 2952-2956 (2013).
14. Fuss, I. J. \& Strober, W. Loss-of-function CARD8 mutation causes NLRP3 inflammasome activation and Crohn's disease. J. Clin. Invest. 128, 1793-1806 (2018).

15. Mitchell, P. S., Sandstrom, A. \& Vance, R. E. The NLRP1 inflammasome: new mechanistic insights and unresolved mysteries. Curr. Opin. Immunol. 60, 37-45 (2019).

16. D'Osualdo, A. et al. CARD8 and NLRP1 undergo autoproteolytic processing through a ZU5-like domain. PLoS ONE 6, e27396 (2011).

17. Duncan, J. A. \& Canna, S. W. The NLRC4 Inflammasome. Immunol. Rev. 281, 115-123 (2018)

18. Ball, D. P. et al. Caspase-1 interdomain linker cleavage is required for pyroptosis. Life Sci. Alliance 3, e202000664 (2020).

19. BioRxiv: Huang, M. et al. Structural and biochemical mechanisms of NLRP1 inhibition by DPP9. 2020.08.13.250241 (2020) https://doi.org/10.1101/ 2020.08.13.250241

20. BioRxiv: Hollingsworth, L. R. et al. Novel mode of filament formation in UPApromoted CARD8 and NLRP1 Inflammasomes. https://doi.org/10.1101/ 2020.06.27.175497.

21. BioRxiv: Hollingsworth, L. R. et al. DPP9 directly sequesters the NLRP1 Cterminus to repress inflammasome activation. https://doi.org/10.1101/ 2020.08.14.246132.

22. Sandstrom, A. et al. Functional degradation: a mechanism of NLRP1 inflammasome activation by diverse pathogen enzymes. Science 364, eaaul330 (2019).

23. Diebolder, C. A., Halff, E. F., Koster, A. J., Huizinga, E. G. \& Koning, R. I. Cryoelectron tomography of the NAIP5/NLRC4 inflammasome: implications for NLR activation. Structure 23, 2349-2357 (2015).

24. Li, Y. et al. Cryo-EM structures of ASC and NLRC4 CARD filaments reveal a unified mechanism of nucleation and activation of caspase-1. Proc. Natl Acad. Sci. USA 115, 10845-10852 (2018).

25. Shen, C. et al. Molecular mechanism for NLRP6 inflammasome assembly and activation. Proc. Natl Acad. Sci. USA 116, 2052-2057 (2019).

26. He, S. \& Scheres, S. H. W. Helical reconstruction in RELION. J. Struct. Biol. 198, 163-176 (2017).

27. Grant, T., Rohou, A. \& Grigorieff, N. TEM, user-friendly software for singleparticle image processing. Elife 7, e35383 (2018).

28. Wang, L., Qiao, Q. \& Wu, H. Understanding CARD tricks in apoptosomes. Structure 25, 575-577 (2017)

29. Lu, A. et al. Molecular basis of caspase-1 polymerization and its inhibition by a new capping mechanism. Nat. Struct. Mol. Biol. 23, 416-425 (2016).

30. Wang, R. et al. Autoinhibition of UNC5b revealed by the cytoplasmic domain structure of the receptor. Mol. Cell 33, 692-703 (2009).

31. Jin, T., Curry, J., Smith, P., Jiang, J. \& Xiao, T. S. Structure of the NLRP1 caspase recruitment domain suggests potential mechanisms for its association with procaspase-1. Proteins 81, 1266-1270 (2013).

32. Jin, T., Huang, M., Smith, P., Jiang, J. \& Xiao, T. S. The structure of the CARD8 caspase-recruitment domain suggests its association with the FIIND domain and procaspases through adjacent surfaces. Acta Crystallogr. Sect. F. Struct. Biol. Cryst. Commun. 69, 482-487 (2013).

33. Wu, B. et al. Molecular imprinting as a signal-activation mechanism of the viral RNA sensor RIG-I. Mol. Cell 55, 511-523 (2014).

34. $\mathrm{Wu}, \mathrm{B}$. et al. Structural basis for dsRNA recognition, filament formation, and antiviral signal activation by MDA5. Cell 152, 276-289 (2013).

35. Gong, Q. et al. Structural basis of RIP2 activation and signaling. Nat. Commun. 9, 4993 (2018).

36. Cheng, T. C., Hong, C., Akey, I. V., Yuan, S. \& Akey, C. W. A near atomic structure of the active human apoptosome. Elife 5, e17755 (2016).

37. Li, Y. et al. Mechanistic insights into caspase-9 activation by the structure of the apoptosome holoenzyme. Proc. Natl Acad. Sci. USA 114, 1542-1547 (2017).

38. Peisley, A., Wu, B., Xu, H., Chen, Z. J. \& Hur, S. Structural basis for ubiquitin-mediated antiviral signal activation by RIG-I. Nature 509, 110-114 (2014).

39. Matyszewski, M. et al. Cryo-EM structure of the NLRC4CARD filament provides insights into how symmetric and asymmetric supramolecular structures drive inflammasome assembly. J. Biol. Chem. 293, 20240-20248 (2018).

40. Holliday, M. J. et al. Structures of autoinhibited and polymerized forms of CARD9 reveal mechanisms of CARD9 and CARD11 activation. Nat. Commun. 10, 3070 (2019).

41. Dick, M. S., Sborgi, L., Rühl, S., Hiller, S. \& Broz, P. ASC filament formation serves as a signal amplification mechanism for inflammasomes. Nat. Commun. 7, 11929 (2016)

42. Robinson, K. S. et al. Enteroviral 3C protease activates the human NLRP1 inflammasome in airway epithelia. Science 370, eaay2002 (2020).

43. Broz, P., von Moltke, J., Jones, J. W., Vance, R. E. \& Monack, D. M. Differential requirement for Caspase-1 autoproteolysis in pathogen-induced cell death and cytokine processing. Cell Host Microbe 8, 471-483 (2010). 
44. David, L. et al. Assembly mechanism of the CARMA1-BCL10-MALT1TRAF6 signalosome. Proc. Natl Acad. Sci. USA 115, 1499-1504 (2018).

45. Pellegrini, E. et al. RIP2 filament formation is required for NOD2 dependent NF-кB signalling. Nat. Commun. 9, 4043 (2018).

46. Kagan, J. C., Magupalli, V. G. \& Wu, H. SMOCs: supramolecular organizing centres that control innate immunity. Nat. Rev. Immunol. 14, 821-826 (2014).

47. Dickson, M. A. et al. Human keratinocytes that express hTERT and also bypass a p16(INK4a)-enforced mechanism that limits life span become immortal yet retain normal growth and differentiation characteristics. Mol. Cell Biol. 20, 1436-1447 (2000).

\section{Acknowledgements}

We thank all members of the Zhong laboratory, Reversade laboratory, and Wu laboratory for their support and help. We acknowledge useful discussions with Dr. Luo Dahai (NTU, Singapore) and Dr. Seth Masters (WEHI, Australia). This work is supported by the Agency for Science, Technology and Research (GODAFIT Strategic Positioning Fund, Bruno Reversade), National Medical Research Council, Singapore (NMRC/ OFYIRG/0046/2017, Franklin Zhong), Concern Foundation's Conquer Cancer Now Award (Franklin Zhong), National Research Foundation, Singapore (Franklin Zhong), Nanyang Assistant Professorship (Wu Bin, Franklin Zhong), MOH-NMRC-OFIRG grant (MOH-000382-00) and Tier II Ministry of Education Research Grant (MOE2016T2-1-010-Wu Bin), as well as Tier I grants (2018-T1-002-010). Bruno Reversade is a fellow of the Branco Weiss Foundation and a recipient of the A*STAR Investigatorship, Senior NRF investigatorship and EMBO Young Investigatorship. Franklin Zhong and Wu Bin are Nanyang Assistant Professors. Franklin Zhong is a recipient of the National Research Foundation Fellowship.

\section{Author contributions}

Q.G. prepared NLRP1 protein samples, conducted all in vitro biochemical experiments, prepared EM samples, collected EM data, and analyzed the results. K.R. and D.T. carried out all cellular experiments and microscopy. C.X. prepared NLRC4 protein samples, conducted imaging processing and computational work. J.Z. prepared a CARD8 sample and conducted computational work. Z.Z.B. prepared ASC proteins conducted biochemical assays related to ASC. P.T.H., H.C.C., and Y.J.T. participated in determining

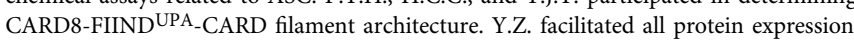
and participated in cloning work. K.R., J.L., W.I.G., and G.W. jointly conducted 3D-SIM experiments and data analysis. K.L. participated in cellular experiments. B.W. and C.X. performed cryo-EM data processing. F.Z., B.W., and B.R. jointly conceived of the project, designed and coordinated the experiments, analyzed the results and wrote the manuscript.

\section{Competing interests}

The authors declare no competing interests.

\section{Additional information}

Supplementary information is available for this paper at https://doi.org/10.1038/s41467020-20319-5.

Correspondence and requests for materials should be addressed to F.L.Z., B.R. or B.W.

Peer review information Nature Communications thanks the anonymous reviewer(s) for their contribution to the peer review of this work. Peer reviewer reports are available.

Reprints and permission information is available at http://www.nature.com/reprints

Publisher's note Springer Nature remains neutral with regard to jurisdictional claims in published maps and institutional affiliations.

(c) (i) Open Access This article is licensed under a Creative Commons Attribution 4.0 International License, which permits use, sharing, adaptation, distribution and reproduction in any medium or format, as long as you give appropriate credit to the original author(s) and the source, provide a link to the Creative Commons license, and indicate if changes were made. The images or other third party material in this article are included in the article's Creative Commons license, unless indicated otherwise in a credit line to the material. If material is not included in the article's Creative Commons license and your intended use is not permitted by statutory regulation or exceeds the permitted use, you will need to obtain permission directly from the copyright holder. To view a copy of this license, visit http://creativecommons.org/ licenses/by/4.0/.

(C) The Author(s) 2021 